\title{
Electrodiffusion phenomena in neuroscience: a neglected companion
}

Leonid P. Savtchenko ${ }^{1}$, Mu Ming Poo ${ }^{2}$, Dmitri A. Rusakov ${ }^{1}$

${ }^{1}$ UCL Institute of Neurology, University College London, Queen Square, London WC1N 3BG, U.K.

${ }^{2}$ Institute of Neuroscience, CAS Center for Excellence in Brain Science and Intelligence Technology, State Key Laboratory in Neuroscience, Chinese Academy of Sciences, 320 Yue Yang Road Shanghai, 200031, China

Correspondence: d.rusakov@ucl.ac.uk 


\section{Preface (100 words)}

The emerging technological revolution in genetically-encoded molecular sensors and superresolution imaging provides neuroscientists with a pass to the real-time nano-world. On this small scale, however, classical principles of electrophysiology do not always apply. This is in large part because the nanoscopic heterogeneities in ionic concentrations and the local electric fields associated with individual ions and their movement can no longer be ignored. Here we review and discuss basic principles of molecular electrodiffusion in the cellular environment of organised brain tissue. We argue that accurate interpretation of physiological observations on the nanoscale requires a better understanding of the underlying electrodiffusion phenomena. 


\section{Introduction}

Over the past decade a large section of experimental neuroscience has been cruising steadily towards the nanoscale. From the single-molecule tracking in live cells ${ }^{1}$ to nano-resolution patch-clamp electrophysiology ${ }^{2}$ to voltage-sensitive dye imaging in sub-micron cellular compartments ${ }^{3}$, the empirical focus on miniscule physiological events in the brain has continuously been sharpening. In the meantime, the fine cellular microenvironment in which such events unfold has been gradually revealed through an advent of the super-resolution imaging methods concentrating on the subcellular architectonics in the live brain ${ }^{4}$.

It thus appears that we are approaching a new frontier in our understanding of neuronal structure and function. The rapid advance into the nano-world of neuronal signalling is set, however, to face a theoretical challenge: accurate interpretation of experimental observations. As the key brain functions rely on electrical activity of nerve cells, translating electrophysiological data into physiological principles has been at the centre stage of neurosciences. In most cases, this gnostic process has relied on the classical theory of electrolytes adapted to the environment of excitable cells many decades ago.

This long-established theory deals with ion fluxes and electric fields at two sides of the cell plasma membrane ${ }^{5}$. Fully consistent with the early experiments in an isolated giant axon of the squid, its main assumptions have been that (a) the space occupied by the electrolyte on either side of the membrane is much larger than the sub-membrane diffusion layers, and (b) there are no external sources of an electric field. However, both assumptions could be largely invalid in organised brain tissue, which is densely packed with electrically active neurons. Indeed, brain cells in situ are constantly exposed to the three main sources of electric field.

First, the macroscopic, time-varying extracellular fields generated by an average net electric current flow due to the spontaneous or behaviour-related (evoked) activities of neuronal assemblies. These macroscopic currents, which are routinely detected by extracellular electrodes as local field potentials, have been a long-rehearsed subject of computational neuroscience because they could provide essential clues to the underlying neuronal activity (see ${ }^{6}$ for a recent review). The present review will therefore touch upon their physiological effects on cells rather than dealing with their mechanistic description. Second, the nonuniform distribution of ion channels and pumps in neuronal membrane, which can produce a net sub-membrane current, steady in resting state and transient during spiking ${ }^{7-9}$. The ensuing local voltage gradients could alter the distribution of signalling molecules and thus influence signal propagation in the local circuitry. Third, in the synaptic cleft microenvironment, receptor-channel currents produce focal perimembrane electric fields directed towards or away from the synapse, both extracellularly and in the cytoplasm. The latter could modify the distribution of synaptic components, in a synaptic type-dependent manner ${ }^{10,11}$.

Because of the relatively small volume tissue fraction occupied by the extracellular space, the magnitude of extracellular electric fields could be quite significant. Theoretical estimates ${ }^{12-14}$ suggest that they are comparable with the externally applied fields that induce documented lateral electrodiffusion of membrane proteins (such as transmitter receptors) in cultured cells ${ }^{15,16}$. Thus, the classical concept of ion movements referring to a large, 
electroneutral medium surrounding nerve cells may produce a biased interpretation of electrodynamic events in nanoscopic cellular compartments, such as synaptic clefts or spine necks ${ }^{17-19}$.

Our understanding of the nanoscale electrophysiology thus may require more adequate insights into small-scale observations, necessitating theories that would account for the submembrane phenomena neglected by the traditional approach (Box 1). In fact, there has been a rapidly growing body of theoretical and experimental studies, albeit mainly outside neurosciences, dealing with the electrolyte dynamics in conditions that are compatible with the brain cell micro-environment ${ }^{20-22}$. The present review aims to explain and illustrate some of these re-emerging concepts and their potential implications for the nanoscale neurophysiology. We will also endeavour to dispel some common misconceptions regarding the nature of the membrane potential while trying not to dwell too much on the wellestablished electrophysiological postulates which the reader can find in numerous textbooks (e.g., ${ }^{23,24}$ ). The present review is by no means intended as an exhaustive guide on electrodiffusion phenomena in nervous tissue, but rather an attempt to discuss theories that are relevant to the electrophysiological phenomena occurring on a small scale, inside and outside cells in the brain.

\section{[H1]Basics of electrolytes}

Brain extracellular fluid is considered a strong electrolyte, i.e. an aqueous solution of (fully) dissociated ionic compounds. In resting conditions (no net ion flow) the electrolyte is assumed to be an electrically neutral medium, with a zero total charge per unit volume $\sum_{i} z_{i} C_{i}=0$ where the sum is over all $i$ th ion species, each having concentration $C_{i}$ and valence $z_{i}$. Electroneutrality is a central principle of the classical electrolyte theory upon which the key electrophysiological derivations have been based. An important and long established assumption here is that the spatiotemporal scale of this theoretical application should be greater than that defined by the two following parameters. One is Debye length $L_{D}$ (1-2 nm range), $L_{D}=\sqrt{\frac{R T \varepsilon_{s} \varepsilon_{0}}{2 F^{2} C}}$ where $R=8.31 \mathrm{~J} \mathrm{~K}^{-1} \mathrm{~mol}^{-1}$ is the gas constant, $F=96485.3 \mathrm{C}$ $\mathrm{mol}^{-1}$ the Faraday constant, $\varepsilon_{\mathrm{s}}$ the relative permittivity or dielectric constant of a solvent, $\varepsilon_{0}$ $=8.854 \times 10^{-12} \mathrm{~F} \mathrm{~m}^{-1}$ the permittivity of free space, and concentration $C$ is defined by $C=\sum_{i} C_{i} z_{i}^{2}$ where $C_{i}$ is the concentration of the $i$ th ion species, and $z_{i}$ is valence. The other parameter is Debye time $t_{D}$ (nanosecond range), $t_{D}=L_{D}{ }^{2} D^{-1}$, where $D$ is the ion diffusion coefficient in the electrolyte ${ }^{25}$. Debye time refers to the Brownian motion in a free medium without external fields. On the scale smaller than the Debye length, spontaneous Brownian motion of individual molecules incessantly breaks medium electroneutrality ${ }^{26}$. Differential ion mobility or the presence of diffusion obstacles and non-electrostatic interactions between ions and cell walls could exacerbate such effects ${ }^{22}$. Such perturbations are thought to normally arise and dissipate with a time constant shorter than the Debye time. 
Importantly, in the bulk of freely moving ions any spontaneous local accumulation of electrical charge should quickly dissipate due to the rapid (electro) diffusion of the oppositely charged ions, thus restoring electroneutrality. Therefore, on a macroscopic scale, over the time intervals exceeding $t_{D}$, a physiological electrolyte is thought to be electrically neutral. This however may change when the theoretical assumptions underlying the electroneutrality principle are no longer valid. The sections below will discuss where and how electroneutrality could be violated and what consequences this may have for our interpretation of empirical observations.

\section{[H1] Extracellular medium}

Macroscopic electric current. The most straightforward case of electroneutrality violation is a net electric current flowing through the region of interest. A current source (usually located on cell membranes) implies a non-zero current density $\mathbf{j}(r)$ across the medium (with co-ordinates $r$ ) (Box 2). This current gives rise to electric field $\mathbf{E}(r)$ present throughout the volume, with its strength depending on local medium conductivity $G$ (Box 2, Equation 1). Thus, at any time point during the active current phase one could find a voltage drop between two points in the medium, implying no electroneutrality. In such cases, a complete set of classical Nernst Planck equations needs to be dealt with, which in many cases lead to robust quantitative estimates ${ }^{27}$.

These theories traditionally work with the classical expression in which conductivity $G$ depends simply on the steady-state ion concentrations (Box 2, Equation 2). The formula is a good approximation in the bulk of brain tissue where a typical electric current alters the steady-state extracellular ion concentration by less than a few percent. However, this approach should be used with caution when applied to nanoscopic volumes, in which modest ion fluxes could alter ion concentrations many-fold and the current density could be spatially heterogeneous ${ }^{13,19}$. In such cases, $G$ will also depend on local electric field strength $E$ (Box 2, Equation 3), thus deviating significantly from the classical approach.

Extracellular space heterogeneity. The electrical conductance of the brain extracellular space (volume conductor) directly affects electrogenic events in nerve or glial cells ${ }^{28,29}$. The unit conductance of the artificial cerebrospinal fluid at $36-37^{\circ} \mathrm{C}$ is $\sim 59 \mathrm{Ohm} \mathrm{cm}$ as reported earlier ${ }^{14}$. However, the actual extracellular conductivity is considerably lower, largely due to the three contributing factors, as follows. Firstly, the extracellular space in the brain occupies only $\sim 20 \%$ of the tissue volume (i.e., tissue porosity $\alpha \sim 0.2)^{30,31}$, which reduces the ion diffusion coefficient hence the effective conductor cross-section accordingly. Secondly, this space comprises tortuous narrow tunnels and clefts surrounding various cellular obstacles $^{32,33}$, with an average macroscopic tortuosity, i.e. an effective increase in the diffusion path compared to a free medium, ranging between $\lambda=1.4-1.6$ (reviewed in ${ }^{31}$ ). Thirdly, even on the nanoscale (no cellular obstacles) the extracellular ion movement is decelerated by $30-50 \%$ compared to a free medium ${ }^{34}$, likely due to microscopic steric hindrance (fixed or mobile molecular obstacles to diffusion) and viscous interactions ${ }^{35}$. An 
additional influence may come from the extracellular matrix, be it a straightforward hindrance to diffusion ${ }^{31}$ or the electrostatic interactions with ions ${ }^{36}$.

The anisotropy and spatial heterogeneity of the extracellular space can lead to non-uniform electric conductivity $G$ and hence the emergence of local voltage gradients, which adds to the complexity of establishing the exact values of electric fields throughout the tissue volume (Box 2 Equations 4-5, Box 2 Figure A-B) ${ }^{37}$. This is likely to affect the interpretation of local field potentials ${ }^{38}$ and the transmembrane potential recordings for experimentally inaccessible areas, such as dendritic spine necks ${ }^{19}$, synaptic clefts ${ }^{14}$ or nanoscopic astroglial protrusions ${ }^{39}$. The medium impedance within these narrow spaces could be significantly higher compared to the bulk tissue, owing to the reduced ion concentration and the increased frequency of ion collisions with microscopic obstacles ${ }^{40}$. An accurate theoretical treatment of the extracellular electrodynamics on the nanoscale is still being developed: the classical volume conductor model with the line source approximation $^{41}$, a detailed Poisson-Nernst-Planck electrodiffusion theory ${ }^{7}$, and the electroneutral approach ${ }^{8}$ provide quantitative insights but appear to diverge when dealing with the immediate membrane proximity. Although beyond the scope of this review, there has also been a concerted effort to understand extracellular electricity in the context of EEG analyses and macroscopic field recordings ${ }^{6,42}$.

\section{[H1]Vicinity of cell membranes}

Membrane surface charges and the adjacent ion layers: It is common knowledge that an electric charge generates an electric field that interacts with other charges. There is a fundamental difference between the spatial distributions of electric fields generated in dielectric media (e.g., dry air) and those generated in electrolytes. In the former case, the electrostatic electric field follows the basic Coulomb's law and, in theory, extends into infinity (Fig. 1A). In electroneutral electrolyte solutions, however, this field extends only within a short distance comparable to the Debye length. Further away than $L_{D}$, and the influence of the field is compensated by mobile ions, making the bulk of electrolyte electroneutral (Fig. 1A). The underlying phenomena can be understood by exploring the nanoscopic region near the phospholipid cell membrane surface, which is negatively charged (Fig. 1B). The charged surface prompts formation of the inner Hemholtz layer of cations, which is immediately adjacent to the membrane, and another layer (more loosely formed, due to a greater effect of Brownian movement), thus forming an electrical double layer (EDL), roughly within the Debye length from the surface (Fig. 1B). The main features of this double layer depend on ion concentrations, the membrane charge density and the thickness of the membrane. The Gouy-Chapman theory proposed a century ago was the first successful attempt to quantify this type of electric double layers at a phase boundary ${ }^{43}$. Its main assumptions (Box 3) enabled robust, if only approximate, theoretical derivations for the electric field near cell membranes. The theory has since been adapted and modified for various sub-membrane scenarios, and its limitations have been explored in detail ${ }^{44,45}$.

More recently, it has emerged that multi-compartmental, Monte Carlo or hybrid (combinedtype) computer models of sub-membrane ion layers could provide a more direct, and arguably more accurate tool to understand ion behaviours in the sub-membrane electrolyte. 
In a typical hybrid model, Monte Carlo computations (which track Brownian motion of individual particles) are carried out within the small volume of interest. At the same time, the flows of particles crossing the volume boundary are continuously recalculated into the flux, field and other parameters representing boundary conditions for the compartmental or analytical model adopted for the large (or infinite) surrounding volume ${ }^{46-49}$.

Computer simulations that account for differential mobility and hydration radii of ions have suggested, for instance, that the bulk $\mathrm{Ca}^{2+}$ concentration of a few $\mathrm{mM}$ could correspond to its levels of up to $50 \mathrm{mM}$ near the cell membrane ${ }^{50}$, in qualitative agreement with earlier analytical estimates ${ }^{51}$. Detailed electrochemical simulations have proposed that in physiological solutions (akin to the cerebrospinal fluid) the medium electroneutrality could be violated within $\sim 2 \mathrm{~nm}$ from the ion-permeable membrane, and over a time interval of up to $40 \mu \mathrm{s}{ }^{52}$. Intriguingly, the latter timescale is comparable with the rising phase of the transmembrane current during action potential generation ${ }^{53}$. Because the sub-membrane extracellular medium is likely to remain heterogeneous over such time intervals, the accurate translation of such measurements into membrane potential could be complicated. With the growing availability of large-scale computation resources required for such models the progress in the area is likely to accelerate.

[H3]Heterogeneous lateral landscapes of membrane surface charge. Because of the nonuniform cellular trafficking and turnover of membrane proteins and phospholipids (including their random fluctuations), the surface charge could be distributed unevenly. This heterogeneity generates lateral, steady-state electrical fields which perturb the classical ion double layer leading to an uneven redistribution of electrolyte ions in the plane of the membrane (Fig. 1C). The resulting profile of the sub-membrane voltage becomes correspondingly heterogeneous (Fig. 1C). A full description of this complex system in steady state involves multiple positive and negative feedbacks, which could be described, with some degree of accuracy, using a set of nonlinear differential equations ${ }^{7,9}$. We note here that the uneven landscape of sub-membrane charges will not only generate laterally heterogeneous voltage, it will also directly affect the transmembrane potential, as explained in the sections below.

[H3]Local channel-mediated current. In another common scenario, a current flows through individual ion channels, generating lateral ion movement along the membrane (Fig. 1D). Given a sufficiently high ion density in the vicinity of the membrane, this flow can exert viscous drag (i.e., pulling along the adjacent liquid layers due to friction) involving both charged and neutral particles such as water molecules (Fig. 1D). In a classical HelmholtzSmoluchowski approximation, just outside the sub-membrane double layer electric field $E$ should prompt the sub-membrane lateral flow with velocity $v$, so that $\nu=-\varepsilon_{r} \varepsilon_{0} E / \eta$,

where $\varepsilon_{0}$ is the permittivity of free space, $\varepsilon_{r}$ is the dielectric constant of the aqueous phase, and $\eta$ is the viscosity of the extracellular medium. The flow rate drops quasi-exponentially with the distance to the membrane, roughly with the Debye length constant. These basic 
effects give rise to the lateral forces acting upon membrane proteins, and may therefore induce their re-arrangement. As explained in the sections below, this phenomenon could contribute to the nanoscopic organisation and dynamics of important membrane specialisations, such as the receptor-protein mobility and clustering in the postsynaptic density.

\section{[H1]Membrane potential: measured vs actual}

[H3]Key assumptions in measuring $V_{m}$. Classically, transmembrane potential $V_{m}$ is determined by the free electrical charges accumulated at the two sides of the cell membrane, in accord with Donnan equilibrium and Goldman-Hodgkin-Katz theory: to calculate $V_{m}$ one has to deal with Nernst-Planck equation and Poisson equation, using the electrolyte composition and membrane permeability, which could be found in textbooks on electrophysiology ${ }^{23,24}$. In healthy neurons, there is an excess of positive charges outside, which results from the different ion composition found inside and outside, from the action of selective ion channels and pumps, and from the presence of impermeable ions in the cytoplasm (ibid).

It is important to note that the sub-membrane voltage generated through local charge accumulation is what essentially controls the voltage-dependent activity of membrane proteins such as ion channels. Their molecular voltage sensors are driven by electric fields in the nanoscopic proximity of the membrane surface (reviewed in ${ }^{54,55}$ ), illustrated in sections below as sub-membrane $V^{*}{ }_{m}$ potential (Fig. 1B-D), rather being directly dependent on the $V_{m}$ readout obtained with electrodes away from the membrane. In patch-clamp practice, however, one inevitably refers to a voltage difference $V_{m}$ between the cell cytoplasm and the bulk of the extracellular medium, as gauged by the current measured in a closed electric circuit (Fig. 2A). The medium around the recording electrodes is routinely assumed to be equipotential, electroneutral, with no electric field present. In fact, the act of recording does violate macroscopic electrolyte electroneutrality, albeit very slightly: this is required to generate the ion current providing voltage readout (Fig. 2A). The case is similar to that of monitoring water pressure in a large vessel where a small leak should serve the purpose, with no detectable effect on the water level (Fig. 2A, inset).

[H3]Non-stationary liquid junction potential. Perhaps the most common empirical factor that could bias macroscopic $V_{m}$ measurements is the liquid junction potential (LJP) ${ }^{56}$. Upon a contact between two electrolytes with distinct ion compositions or mobility, excess charge emerges at the interface, generating a local electric field (Fig. 2B). In the simplest case of two binary electrolytes with ion concentrations $C_{1}$ и $C_{2}$, it is given by the Henderson equation $\Delta \varphi=\frac{u_{+}-u_{-}}{u_{+}+u_{-}} \frac{R T}{F} \ln \left(\frac{C_{2}}{C_{1}}\right)$ where $\mathrm{u}_{+}$and $\mathrm{u}_{-}$are the ion mobility. The formula assumes electroneutrality (no strong current) inside and outside the electrode. Three LJP types have classically been considered ${ }^{57}$ : same salts - different ion concentrations, different salts -similar ion concentrations; different salts- different ion concentrations. Whilst LJP can be estimated with various online calculators (e.g., 
http://web.med.unsw.edu.au/phbsoft/LJP_Calculator.htm) based on known ion concentrations, recent studies emphasise that the LJP is not in steady-state ${ }^{27,58}$. During the continued dialysis and solution mixing, this interface could expand away from the electrode, with the boundary charge density decreasing and the ion concentrations partly equilibrating (Fig. 2B).

[H3]Distortions invisible to patch clamp. As pointed out above, one of the key factors affecting $V^{*}{ }_{m}$ on the nanoscale is the distribution and screening (cancelling out by the nearby opposite charges) of electric charges carried by molecules at or near the cell membrane surface, which contribute to the Gouy-Chapman electrical double layer (EDL) 59 . If the surface charge landscapes carried by the inner and the outer membrane leaflets are roughly mirrored, the net field generated by EDLs across the membrane does not distort the sub-membrane local $V^{*}{ }_{m}$ or pipette-measured macroscopic $V_{m}$ (Fig. 2C, Symmetric case). Whilst this scenario is thought to be relatively common, an asymmetric distribution of neutral lipid molecules, in particular sphingomyelin or cholesterol, in one of the two membrane leaflets can modify the surface charge and thus the local $V^{*}{ }_{m}$ potential ${ }^{60,61}$ (Fig. 2C, Asymmetric leaflets). The latter in turn would shift accordingly the activation curve of voltage-sensitive membrane proteins that occur nearby.

[H3]Divalent ion screening. An even more prominent influence on $V^{*}{ }_{m}$ may come from the variable ratios of divalent and monovalent cations in the solution. Divalent cations $\left(\mathrm{Ca}^{2+}\right.$, $\mathrm{Mg}^{2+}$, etc.) have a greater charge with a smaller effective radius and therefore provide more efficient screening of negative surface charges (Fig. 2C, Divalent ion screening). When the concentration of divalent ions changes, inside or outside the cell, the overall chargescreening efficiency may also change and thus alter the voltage drop across the membrane. This could have a substantial effect on voltage-gated ion channels that sense local $V^{*}{ }_{m}$, and thus on cell excitability ${ }^{62}$, not necessarily affecting patch-clamp measurements of $V_{m}$. Such phenomena could be activity-dependent: for instance, neuronal discharges could transiently lower local extracellular $\left[\mathrm{Ca}^{2+}\right.$ ] (from its normal level of 1.5-2.0 mM) as a result of $\mathrm{Ca}^{2+}$ entry ${ }^{63}$ or indirect consequences of $\mathrm{Na}^{+}$entry ${ }^{64}$ into neurons. This in turn would have a disproportionately large effect on sub-membrane $\left[\mathrm{Ca}^{2+}\right]$ (which could reach a $50 \mathrm{mM}$ range due to the favourable conditions for $\mathrm{Ca}^{2+}$ accumulation near the negatively charged membrane, compared to $\mathrm{Na}^{+}$or $\left.\mathrm{K}^{+}\right){ }^{65}$. Indeed, early calculations ${ }^{66}$ suggested that $1 \mathrm{mM}$ of a divalent ion added to $100 \mathrm{mM}$ electrolyte could shift membrane potential by $\sim 26 \mathrm{mV}$ due to the screening effect. With these considerations in mind, classical studies of ion channels have routinely documented voltage-dependent channel properties over a range of divalent ion concentrations ${ }^{67-69}$. It would seem important for the electrophysiological observations of today to account for such phenomena.

[H3]Biochemical and metabolic modification of surface charges. Finally, a potentially crucial contributor to $V^{*}{ }_{m}$ could be the biochemical or metabolic alteration of the membrane surface charge density through interaction with other molecules. One important example is the changes in the membrane charge landscape through sialylation by neuraminidase (an enzyme that cleaves sialic acids): this reaction is a common part of molecular signalling cascades in the brain. Sialylation can cause a large depolarising shift in the activation curve 
of voltage-gated sodium channels, also altering the spiking threshold of the neuron, without any change in the patch-clamp recorded membrane voltage $V_{m}$ in hippocampal pyramidal neurons $^{70}$. This is because the removal of negative surface charges increases (hyperpolarises) $V^{*}{ }_{m}$, with little effect on $V_{m}$ (Fig. 2C, Neuraminidase charge cleavage), thus making it more difficult for the external depolarising current to activate sodium channels. Modification of voltage-gated channels through sialylation could be a common phenomenon across various types of excitable cells ${ }^{71}$, and it might potentially explain the role of extracellular matrix proteins in regulating cell excitability and synaptic plasticity ${ }^{72-}$ 74. The aggregation of membrane proteins may also result in local changes in the surface charge density which in turn would affect protein function. In a key demonstration of such phenomena, the burst duration of acetylcholine (ACh) receptor-channel kinetics was significantly prolonged in the muscle cell membrane when ACh receptors were induced to form a stable aggregate in the membrane after an exposure to a physiologically relevant extracellular electric field $\left(10^{2}-10^{3} \mathrm{~V} / \mathrm{m}\right)^{75}$.

\section{[H1]Intracellular space}

General notes. It has long been acknowledged that in thin dendrites and dendritic spines of nerve cells the dynamics of the ion concentration and the membrane potential follow the Nernst-Planck equation, rather than the cable equation ${ }^{76}$. This is because in small volumes the ion concentration cannot be assumed constant, which is a key prerequisite of the cable equation. However, this valid correction has not been widely used, most likely owing to the complex numerical calculations involved and the difficulty of measuring local membrane potential. Subsequently, there had been several attempts to improve the solution accuracy for small spaces by introducing fractional Nernst-Planck equations combined with the corresponding fractional cable equations, to model ion electrodiffusion in nerve cells ${ }^{77,78}$. Whilst the cable equation still provides a well-trodden path to study the cell membrane electrodynamics efforts are being made at adopting the Nernst-Planck equations more widely, to model electrogenic processes in neurons and glia more accurately ${ }^{79,80}$.

[H3]Cell-impermeable anions (CIAs) and perturbation of electroneutrality. The cell cytoplasm hosts a variety of CIAs - proteins, hydrogen phosphate groups, sulphates, other organic macromolecules - that remain negatively charged at intracellular $\mathrm{pH}^{81}$. These macromolecules are surrounded by cytoplasm cations, thus perturbing the cytoplasm electroneutrality, at least on the nanoscale (Fig. 3A). Such perturbations could form local ion layers, and correspondingly, electric fields that could affect local molecular signalling mechanisms.

Interestingly, the effective concentrations of the negative charges carried by CIAs could reportedly reach concentrations as high as $100-200 \mathrm{mM}^{82}$. It has recently been suggested that the actively regulated intracellular redistribution of such protein-associated CIAs could effectively control the intracellular chloride concentration thus imparting on GABAergic transmission ${ }^{83}$. This idea was based on the simple postulate that, to maintain the net sums of charges inside and outside the cell, the redistribution of CIAs in the cytoplasm could 
effectively displace other anions, mostly chloride. This interpretation was later challenged by providing alternative, physiologically relevant explanations of the original experimental observations ${ }^{84-86}$, but whether it was, in principle, biophysically plausible has remained uncertain.

Assuming that individual CIAs are evenly distributed in the aqueous cytoplasm, 100-200 $\mathrm{mM}$ corresponds to an average volume density $n \sim 0.1 \mathrm{~nm}^{-3}$, with an average nearestneighbour distance $a=\left(\frac{3}{4 \pi n}\right)^{1 / 3}$ of $\sim 1.35 \mathrm{~nm}$. This distance is comparable with the Debye length for 100-200 mM strong electrolytes at physiological temperatures. In this case the CIAs could, in theory, displace chloride ions, as there will be limited space left for forming screening layers and maintaining a free electroneutral (chloride-containing) medium in between individual CIAs. However, this simplified calculation assumes that individual CIA charges and small cytosol cations (mainly chloride) form an ideal-gas-type thermodynamic equilibrium, which is unlikely to be case, as explained below.

[H3]Small volume occupied by CIAs should leave enough room for free anions. In fact, detailed biophysical studies of the intracellular milieu indicate that macromoleculeassociated CIAs are grouped in polymer-like structures forming an intracellular hydrogel 'matrix' ${ }^{87-89}$ which is intrinsically sensitive to osmolarity and $\mathrm{pH}^{90}$. While featuring a high surface charge density (nominally exceeding $200 \mathrm{mM}$ on the nanoscale) such structures appear to occupy only a small fraction of the cytosol volume ${ }^{91}$, thus permitting relatively free diffusion of small molecules in between ${ }^{92}$. Similar logic applies to the effects of intracellular organelles bearing a surface charge: for instance, in physiological conditions, actin filaments of the cytoskeleton bear the charge at a linear density of $\sim 4 \cdot 10^{3} e / \mu \mathrm{m}^{93}$. It has recently been estimated ${ }^{94}$ that in such cases the charge-screening ion layers are formed around the densely accumulated surface charges while the bulk of intracellular electrolyte remains in an equilibrium with free-diffusing ions such as chloride (Fig. 3B). The 'cloud' of ions surrounding such structures serves as a Debye shield forming a narrow cable-like arrangement. The prevalence of an electroneutral, mostly equilibrated electrolyte explains the relatively unrestricted diffusion of various signalling molecules in the cytosol.

It appears therefore that, unless a local displacement of chloride by CIAs occurs in the immediate intracellular vicinity of chloride channels, the driving force for chloride is unlikely to be influenced by CIAs (Fig. 3B). Nonetheless, whilst the latter scenario seems more plausible than the direct effect of CIAs on chloride currents ${ }^{83}$, the exact features of ionic landscapes inside the neuronal cytoplasm remain incompletely understood ${ }^{87}$. Because slowly-moving or immobile proteins or intracellular organelles could thus affect local electro-neutrality, the mechanism of intracellular voltage regulation appears complicated, involving multiple time scales.

\section{[H1]Narrow extracellular spaces}

[H3]Neurotransmitter electrodiffusion in the synaptic cleft. The most intense extracellular fields generated endogenously in the nervous system seem to occur near excitatory synapses. 
Either spontaneous or evoked release of transmitters (glutamate or ACh) could trigger inward membrane currents through the postsynaptic clusters of transmitter receptorchannels, with the focal extracellular fields pointing towards the synaptic cleft and the cytoplasmic fields pointing away from the synapses. Thus it has long been noted that when a synaptic inward current flows through receptor-channels in the middle of a narrow synaptic cleft, this could form a significant lateral voltage drop across the cleft ${ }^{12,95}$. In the bulk of the cleft volume (away from the membrane), ion diffusion could be described using classical equations of electrodiffusion (Box 1, example). Such focal electric fields may serve three different purposes at the synapse: formation, maintenance, and activity-dependent plasticity.

The relatively small ratio of the extracellular versus cytoplasmic volume (0.1-0.2) dictates a much higher extracellular field than cytoplasmic field. Estimates based on the synaptic current measurements point to the electric fields peaking in excess of $10^{2} \mathrm{~V} / \mathrm{m}$ that may exist at the synaptic cleft ${ }^{96}$. In the case of small central synapses equipped with synaptic receptorchannels of AMPA and NMDA types, detailed Monte Carlo modelling suggests that the sodium current flowing from outside could give rise to a local field reaching $\sim 10^{4} \mathrm{~V} / \mathrm{m}$ inside the cleft ${ }^{13,14}$.

Whilst this strong field can only slightly perturb local pre-equilibrated concentrations of the prevailing extracellular ions, such as sodium or potassium ${ }^{48}$, experiments have indicated that it can accelerate diffusional escape of glutamate molecules (negatively charged at physiological $\mathrm{pH}$ ) two-three-fold following their release and receptor activation ${ }^{48,97}$ (Fig. $4 \mathrm{~A}, t o p$ ). When glutamate release happens to coincide with the postsynaptic spike (i.e. sharp reversal of the current and hence of electric fields), electrodiffusion slows down glutamate escape thus boosting activation of local high-affinity receptors (Fig. 4A, middle; note that the bulk of high-affinity glutamate uptake occurs further away, outside the cleft). Such electrodiffusion-driven activation of the otherwise 'silent' high-affinity receptors could trigger synaptic plasticity, as shown for perisynaptic metabotropic glutamate receptors at cerebellar synapses ${ }^{48}$ (Fig. 4A). In studying such phenomena, a routine assumption has been that external electric fields have no effect on the ligand-receptor binding per se. Whilst this notion has been supported, at least in part, by detecting no effect of intra-cleft fields on receptor currents activated by electrically neutral GABA ${ }^{97}$, the net influence of electric fields on receptor binding requires further studies.

[H3]Protein migration in the synaptic cleft under electric fields. It has long been proposed that focal currents generated by the nascent synaptic connections give rise to electric fields that may help to recruit plasma membrane and cytoplasmic components for the establishment of pre- and postsynaptic specializations, in a positive-feedback manner ${ }^{98}$. One could assess the overall effect of transient electrostatic forces on charged membrane proteins by calculating the molecule's velocity $v$ in a viscous membrane medium. As the viscous resistance depends highly supra-linearly on $v$, the velocity value quickly stabilises, and becomes linearly proportional to the electric field in accord with the classical relationship:

$v=D\left(\frac{F}{R T}\right) f_{e}=D\left(\frac{z F}{R T}\right) E$

where $f_{e}$ is electrostatic force, $E$ is electric field, $D$ is intra-membrane diffusion coefficient, $z$ is valence. With the $E$ value reaching $\sim 10^{4} \mathrm{~V} / \mathrm{m}$ inside the cleft ${ }^{14,97}$, this expression predicts 
a force of $\sim 10^{-15} \mathrm{~N}$. Given the expected lateral diffusion coefficient for major synaptic receptors (AMPA and NMDA type) of $\sim 50 \mathrm{~nm}^{2} / \mathrm{ms}\left(5-100 \mathrm{~nm}^{2} / \mathrm{ms} \mathrm{range}^{99,100}\right), v$ will be in the range of $\sim 50 \mathrm{zm} / \mathrm{s}$. There is a paucity of data shedding light on the net electrostatic charge carried by extracellular compartments of synaptic receptors, let alone the uncertainty about the relationship between such charges and the immediate molecular environment. One protein structure study has estimated that NMDA receptors could carry an extracellular positive surface charge in the region of $z=20-30{ }^{101}$. This would correspond to $v=1-1.5$ $\mathrm{nm} / \mathrm{ms}$ predicting that the 100-200 ms inward current (characteristic time for the synaptically activated opening of NMDA receptors) could, in theory, move NMDA receptors towards the peak current source by many tens of nanometers (Fig. 4B). Although the postsynaptic receptor clustering could be triggered by other mechanisms, such as Brownian diffusion with traps ${ }^{102}$ or the presence of co-operative membrane surface binding sites ${ }^{103}$, the faster time scale provided by electrodiffusion remains an attractive proposition for rapid receptor re-arrangement.

After the synapse matures, turnover of synaptic component (exchange of newly synthesised proteins with the old ones) in the plane of plasmalemma and in the cytoplasm ${ }^{104,105}$ may be facilitated by the constant presence of these focal fields generated by spontaneous or evoked transmitter release. Similarly, in the course of use-dependent plasticity, such as long-term synaptic potentiation induced by intense neurotransmitter release ${ }^{106}$, electric fields might enhance recruitment of synaptic components such as synaptic receptors via lateral migration 10,107,108 (Fig. 4B).

[H3]Other narrow spaces. Similarly to the case of synaptic clefts, the narrow space between cell walls could lead to a high local current density and strong electric field, thus breaching canonical assumptions of classical electrophysiology. In this context, one structure that is central to signal communication in the brain deserves particular attention, the thin neck of dendritic spines. Here we refer the reader to a recent review exploring electrodiffusion phenomena pertinent to the electric signal propagation through the spine neck ${ }^{19}$. Detailed theoretical studies have predicted that within such small spaces the local membrane curvature $^{109}$ and the surface-volume relationships ${ }^{110}$ can also be important in modulating the membrane voltage. Another common scenario involving narrow-space electrodiffusion phenomena arises when an action potential is propagating along an axon that is closely surrounded by neighbouring cellular structures. In this case, the lateral current gives rise to a non-zero electric field inducing lateral electrodiffusion flow of charged molecules within and outside the axonal membrane, thus perturbing the double layer composition ${ }^{111}$. Thus, the microscopic environment of non-myelinated axons could in theory affect spike propagation and local molecular traffic in a mechanistic fashion: future experiments should reveal how exactly this mechanism acts.

[H3]Does the theory work with only a few molecules? In some cases, the number of diffusing particles within the volume of interest is so small that the spontaneous fluctuations in their number (due to their Brownian movement) are comparable with or exceed the average value normally represented by the concentration parameter. For instance, the 
physiological concentration of $50 \mathrm{nM}$ characterising free $\mathrm{Ca}^{2+}$ in a dendritic spine head or in a nanoscopic astrocyte process corresponds to only a few ions ${ }^{112,113}$. Although in that particular case the small number reflects a dynamic equilibrium between free and bound $\mathrm{Ca}^{2+}$ ions (the latter being 100-1000 times more prevalent), Monte Carlo simulations would be required to predict stochastic fluctuations arising from the movements of individual molecules in such cases. It has long been understood that the stochastic processes characteristic for such small volumes (i.e., volumes in which only a small number of molecules are present) could play a critical role in molecular dynamics. It has been argued that the thermal noise and the nanoscopic size of the ions affect the amplitude ${ }^{114}$ and the variability of the electric current passing through ion channels ${ }^{115,116}$. The finite size of ions inside the sub-membrane double layers can modify the interaction between two neighbouring cell membranes if the inter-membrane clearance is comparable to the thickness of electrical double layers ${ }^{117}$. The variability of the effective ion concentration influences the sensitivity of membrane receptors, thus setting the limit at which the receptor binding kinetics could be evaluated ${ }^{118}$. In the majority of such cases, there is little choice but to explore such events using Monte Carlo simulations that recapitulate stochastic movements of individual particles.

\section{[H1]Membrane proteins and external fields}

[H3]Lateral electrodiffusion and electroosmosis of membrane proteins. The phenomenon of lateral electrodiffusion of protein molecules in the plasma membrane in response to extracellularly applied electric field has long been known ${ }^{15,119}$. In cultured embryonic muscle cells, application of a steady extracellular field resulted in the accumulation of acetylcholine (ACh) receptors towards the cathodal side of the cell, and in the formation of immobile ACh receptor clusters in the muscle membrane ${ }^{16}$. Interestingly, the removal of cell surface sialic acid with neuraminidase, which reduces the cell surface negative charge, reversed the direction of electrodiffusion ${ }^{16}$. This result is consistent with the idea that the electrodiffusion of membrane proteins is driven not only by "in situ" electrophoresis, but also by electro-osmosis, in which the flow of cell surface positively-charged counter-ions provides the dominate force in dragging negatively charged proteins towards the cathode ${ }^{120}$ (as in Fig. 1D, also illustrated in ${ }^{121}$ ). Furthermore, a unipolar pulsed electric field could also induce electrodiffusion towards the cathode ${ }^{122}$, suggesting a cumulative effect of the field.

Modulation of cell membrane potential located in external fields. Besides altering the distribution of membrane proteins, extracellular fields also modulate the local membrane potential, with a depolarising effect on the cathode-facing side of the cell. This membrane potential modulation is particularly pronounced for long processes that are aligned along the direction of the field, since the voltage drop across the plasma membrane at two ends of the process are much larger than that across the cell soma. This local membrane potential modulation will in turn affect the extent of activation of voltage-dependent ion channels. It has been shown that a focal uni-directional pulsed field causes a galvanotropic turning of the axon growth cone towards the current sink generated by a glass microelectrode ${ }^{123}$. This 
effect on the growth cone could be attributed to the asymmetric local elevation of $\mathrm{Ca}^{2+}$ at the growth cone caused by electric field across the growth cone ${ }^{124}$.

\section{[H1]Concluding remarks}

Numerous theoretical studies and accumulated experimental evidence leave us in little doubt that endogenous neural activities generate strong heterogeneous electric fields within the tissue, particularly in the extracellular space. Such effects become particularly significant in the restricted spaces, such as small cellular compartments or inside the synaptic cleft. In the latter case, electric fields generated by synaptic currents could directly affect excitatory transmission and its use-dependent changes. To what extent such fields produce direct electrokinetic effects on neural components at a level that could in turn modulate neural functions in a broader context requires further studies. Demonstrating the physiological significance of the electrodiffusion phenomena in real-life scenarios remains a critical issue.

The experimental verification of the causality between electrokinetic effects and neural functions is not straightforward, mainly because such effects are often inseparable from other chemical processes associated with electrical activity, but also because direct experimental probing on the nanoscale has severe limitations. For example, demonstrating a direct action of focal electric fields on synaptic receptors would require experimental elimination of the focal fields only, without affecting other chemical processes associated with the synaptic activity, such as $\mathrm{Ca}^{2+}$ influx and kinase activation. Nevertheless, the ubiquitous physical presence of endogenous electric fields and the inevitable physical actions of these fields on neuronal components represent a subcellular aspect of the nervous system that deserves more attention and appreciation. By using the movement of electrolytes as a means for communication in the nervous system, the process of evolution could have also taken advantage of various electrokinetic phenomena such as electrodiffusion to serve useful neural functions. With the ever increasing spatiotemporal resolution of real-time experimental observations in nerve and glial cells, including local electrodiffusion phenomena in the data interpretation is expected to become a routine. 


\section{Box 1 Nanoscale neurophysiology: classical theories and where they may stumble}

A partial list of issues that nanoscale neurophysiology could encounter when referring to the traditional theories.

1. The electric potential of the membrane approached closely by another membrane could differ from the potential of the membrane facing a large free volume. This is because inside a narrow space the electroneutrality condition could be breached and the medium conductance (hence local potential) could be non-uniform, depending on fluctuations in local current and ion concentration.

2. In organised brain tissue the local extracellular space often has a smaller cross-section and therefore higher impedance than does the intracellular lumen. In this case lateral propagation of membrane potential will depend mainly on the extracellular, rather than intracellular, medium, in contrast to the classical view.

3. With a relatively high lateral resistance of narrow extracellular space, local electric fields could be strong enough to influence lateral movement of charged molecules in the plasma membrane. These electrophoretic effects could therefore influence particle traffic in neuronal membranes.

4. Similarly, strong lateral electric fields near charged membranes also give rise to an electroosmotic flow of counter-ions, which could drive lateral migration of charged and electrically neutral molecules along the plasma membrane.

5. Ion channels sense the local charge density (electric field) rather than the average transmembrane potential recorded by a patch electrode. Whilst in basic conditions the charge density scales with the measured transmembrane potential, this relationship could be breached by the non-uniform accumulation of surface charges or by the heterogeneity in membrane (lipid) composition. In such cases experimental readout of voltage-dependent channel kinetics could be heavily biased.

6. Most classical theories are based on the quasi-static approximation of Maxwell's equations which assume no effect of magnetic fields and no uncompensated charges present in the medium. Both assumptions could be invalid on the nanoscale.

The classical formulism of electrodiffusion could be illustrated by the diffusion of charged particles inside a flat narrow space with a radial electric field (for instance, the synaptic cleft with a receptor current directed towards the centre). This diffusion follows the Nernst-Plank equation in conditions of rotational symmetry:

$$
\frac{\partial C}{\partial t}=D\left(\frac{1}{r} \frac{\partial}{\partial r}\left(r \frac{\partial C}{\partial r}\right)+\frac{z F}{R T} \frac{1}{r} \frac{\partial}{\partial r} r C E\right)
$$

where $r$ is the radial co-ordinate, $C(r, t)$ stands for ion concentration, $D$ is the diffusion coefficient, $z$ is valence, $F$ is the Faraday's constant, $R$ is gas constant, $T$ is temperature, and $E(r, t)$ is the electric field strength. In a steady state approximation (concentrations and currents remain unchanged over the measurement period), $E(r)$ could be expressed using the 
medium resistivity $R$, the cleft height $h$ and electrical current $I(r)$ flowing through the cleft volume:

$$
E(r)=\frac{d V}{d r}=-\frac{I(r) R}{2 \pi h r}
$$

Combining equations (1) and (2) gives:

$$
\frac{\partial C}{\partial t}=D\left(\frac{\partial^{2} C}{\partial r^{2}}+\frac{1}{r} \frac{\partial C}{\partial r}\left(1-\frac{z F}{R T} \frac{I R}{2 \pi h}\right)\right)
$$

Here the current is calculated from

$$
I=2 \pi \int_{0}^{\infty}\left(V(r)-V_{c}\right) g(r) r d r
$$

where $g(r)$ is the radius-dependent conductance (e.g., due to synaptic receptor channel opening), and $V_{c}$ is the conductance reverse potential.

Note that concentration $C$ is an averaging quantity, which is relevant for large numbers of molecules, when the fluctuations in their numbers arising from their stochastic movement or chemical reactions are negligible. When the numbers of diffusing molecules are small, the effects under study could depend on the number variability rather than on its average. In such cases it would be natural to use Monte Carlo (single-particle tracking) simulations, rather than analytical tools, to evaluate the electrodiffusion phenomena involved ${ }^{48,97}$.

\section{Box 2 Basic relationships between electric field, current, and conductance}

The field strength $\mathbf{E}(r)$ generated by the external current in an electrolyte is

$$
\mathbf{E}(r)=\frac{\mathbf{j}(r)}{G}
$$

where $\mathrm{j}$ denotes current density, $r$ is the spatial coordinate, and the conductivity of (strong) electrolyte $G(r)$ is classically given by:

$$
G=\frac{F^{2}}{R T} \sum_{n=1}^{i} D_{n} z_{n}^{2} C_{n}
$$

where $z_{n}, C_{n}$, and $D_{n}$ are the valence, concentration and diffusivity, respectively, of the $n$th ion species, respectively, and $T$ is temperature.

Expression (2) is key to the traditional interpretation of electrophysiological recordings: it assumes overall electroneutrality and thus constant electrolyte conductivity $G$ which depends simply on the bulk concentration of ions. However, when electric fields perturb ion concentrations $C_{n}$ and redistribute local charges (particularly within narrow spaces) electroneutrality will be violated. In such cases, conductivity $G$ (Figure, A; green shade scale) will depend on electric field strength $E$ (Figure, A; blue arrows indicate direction and 
am increased strength where $G$ is higher). This will determine local current density $\mathbf{j}$

(Figure, A; red arrows indicate direction and a reduced magnitude where $G$ is lower). Thus, when the space is heterogeneous, $G$ will depend on the spatial co-ordinate $r$ :

$$
G(r, E)=\frac{F^{2}}{R T} \sum_{n=1}^{i} D_{n} z_{n}^{2} C_{n}(r, E)
$$

In this context, it is important to note that fluctuations in the local concentration of any ion should trigger concentration changes in all other ions as the system always drives towards restoring local electroneutrality. Also, the classical linear dependence between medium conductivity and ion concentration assumes that ion concentrations are sufficiently low to neglect direct molecular interactions. Increasing ion concentrations and thus inter-ion interactions could disproportionately decrease the medium conductivity ${ }^{125}$.

In a general case of conductivity involving anisotropic extracellular media ${ }^{37,126}$, field and current vectors $\mathbf{E}$ and $\mathbf{j}$ are not necessarily collinear (Figure, B). Canonically, their relationship can be described as

$$
\mathbf{j}_{\mathrm{i}}=\mathbf{G}_{\mathrm{ik}} \mathbf{E}_{\mathrm{k}}
$$

where conductivity $\mathbf{G}_{\mathbf{i k}}$ is a second-rank tensor, which scales and rotates $\mathbf{E}$ with respect to j. The current in this case will flow along the three-dimensional path of lowest electrical resistance (Figure, B; red arrows indicate direction of $\mathbf{j}$, which is rotated with respect to the direction of $\mathbf{E}$ ). It is important to note that, according to the essentials of thermodynamics, there cannot be 'residual' ion current that occurs without electric field in electrolyte (current $\mathrm{j}_{0}$ so that $\mathbf{j}_{\mathbf{i}}=\mathbf{G}_{\mathbf{i k}} \mathbf{E}_{\mathbf{k}}+\mathbf{j}_{\mathbf{0}}$ ) as this would violate the principle of increasing entropy ${ }^{126}$.

\section{Box 3. Key assumptions of Gouy-Chapman theory of the electrical double layer}

- Ions in the solution are modelled as point charges thus neglecting their physical dimensions and arising singularity

- The solvent is considered a dielectric continuum with dielectric constant $\varepsilon$

- The average concentration of ions at a given point reflects the average the electrostatic potential at that location, in accord with the Boltzmann distribution, which is a probability function $F(S)$ of the stochasticallybehaving particles being in a certain state $S$ in a multi-particle system: $F(S) \sim \exp (-\Psi / k T)$ where $\Psi$ is state-designated energy, $k=1.38 \times 10^{-23} \mathrm{~m}^{2}$ $\mathrm{kg} \mathrm{s}^{-2} \mathrm{~K}^{-1}$ is Boltzmann constant, and $T$ is Kelvin temperature.

Approximations routinely employed in derivations exploring sub-membrane ion layers (largely based on the Poisson-Boltzmann theory):

- The membrane is treated in a continuum limit as an interface with a contiguous surface charge distribution, with no point or discontinuous features such as ion channels. 
- The electric potential and the ion charge densities are described by continuous variables.

- Non-electrostatic interactions, such as Van der Waals interactions, are neglected.

- Membrane boundaries or curvature are not considered.

Common approximations and assumptions in theories dealing with macroscopic extracellular electric fields

- Extracellular space is homogenous and isotropic, with a constant unit conductance.

- Ions move in the extracellular space without boundary effects, friction or steric hindrance.

- In baseline conditions, the principle of electroneutrality is hold throughout the medium so free particle diffusion is assumed.

- The effects arising from water molecule movement are neglected.

- The condition of electroneutrality is maintained at infinity in the equations. 


\section{FIGURE LEGENDS}

\section{Figure 1. Electric charges and their fields in brain tissue: basic principles and two common deviations from common electrophysiological postulates.}

A: Electrostatic electric fields generated by a local (point) charge and a charged plane, in either vacuum (dielectric medium, top) or electrolyte (bottom) are shown, where colour intensity illustrates field strength. Whereas the field (depicted by arrows) in a vacuum extend into infinity, in electrolytes they are highly localised.

B: Ion distributions and local voltage profile near a negatively charged phospholipid cell membrane surface; inner Helmholtz layer (a layer of cations lined up next to the negatively charged membrane) and Gouy-Chapman double layer ( $G$ - $C$, includes the Helmholtz layer and a relatively loose layer of anions adjacent to it) are indicated. $V^{*}{ }_{m}$ depicts voltage profile (arrow indicates voltage scale) at a short distance from the membrane (dotted line, not to scale) were signalling proteins such as ion channels may sense local electric fields (see below). The $V^{*}{ }_{m}$ profile shows a canonical case of the nearly-evenly charged membrane is illustrated.

C: Heterogeneity of sub-membrane ion distribution and local voltage due to excessive membrane charges (carried by either phospholipids or membrane proteins; shown by additional blue ovals). The uneven occurrence of cations (red) and anions (blue) reflect the variable density of local electric fields and hence the greater variability in sub-membrane voltage $V^{*}{ }_{m}$ compared with panel B.

D: Heterogeneity of sub-membrane ion distribution and local voltage due to ion channel currents; red arrowheads, current direction (ion channel is shown); black arrow depict drag forces exerted by the cation current flow: these tend to tow alongside the sub-membrane ion layers.

\section{Figure 2. Patch-clamp measurements of membrane potential: first principles.}

A: Simplified diagram of a standard patch-clamp configuration; membrane potential is depicted by the excess positive charge outside the cell. Taking a measurement of current $I(V)$ involves a breakdown, albeit almost negligible, of the medium electroneutrality. Inset, a water pressure measuring analogy of $V_{m}$ measurements: the rate of a small leak can be used to evaluate water pressure (height of potential) in a large volume, without affecting the water level.

B: Diagram illustrating non-stationary liquid junction potential $\left(V_{L}\right)$ at an interface of pipette solution (grey) and bath solutions (blue, no cells present). When the two solutions have distinct ion compositions and/or ion mobility, this gives rise to a trans-interface electric field (shown by the accumulation of two different ion types at the solution interface). During the dialysis the boundary can expand and / or dissipate thus reducing the liquid junction potential to a new value $V_{L}{ }^{*}<V_{L}$. 
C: Common distortions of transmembrane potential which are invisible in patch-clamp configuration. A schematic depicting the cell membrane bilayer and sub-membrane ion layers; $V$ (thick red line), the 'true' voltage profile across the membrane; $V_{m}$, transmembrane voltage measured in patch-clamp configuration, away from the membrane; vertical dotted green lines, the approximate sub-membrane locality that primarily affects the channel voltage sensor; $V^{*}$ (green), transmembrane voltage sensed by ion channels at that locality (as in Fig. 1B-D).

Symmetric (canonical) case: Because sub-membrane electric fields quickly dissipate away from the membrane, measured membrane potential $V_{m}$ is likely to be somewhat larger than the sub-membrane $V^{*}{ }_{m}$ sensed by receptors and channels. Asymmetric leaflets: The effect of the two asymmetrically charged membrane (bilayer) leaflets: the inside leaflet is shown more negatively charged, shifting the $V$ profile (red line) accordingly. This increases the $V^{*}{ }_{m}$ compared to the canonical case, with little effect on $V_{m}$ measured by the patch pipette, the later thus underestimating $V^{*}{ }_{m}$. Divalent ion screening: The effect of the divalent ion (red ovals) screening of the membrane charge; this extends the characteristic intramembrane $V$ profile (red line) outside the membrane leaflet, again, increasing $V^{*}{ }_{m}$ with no effect on $V_{m}$. Neuraminidase charge cleavage: The effect of metabolic activity (the negative charge cleavage with neuraminidase; white ovals depict loss of negative charge); again, this increases the local transmembrane voltage drop $V^{*}{ }_{m}$ with little effect on pipette readout $V_{m}$.

\section{Figure 3. The effect of membrane-impermeable intracellular anions on membrane ion} exchange.

A: A schematic depicting large cell impermeable anions (CIAs, blue ovals) surrounded by a layer of cations; the local excess of positive change and adjacent anions means that the local lateral voltage profile $\left(V^{*}\right)$ is not uniform but has the corresponding local deviation(s).

B: A schematic depicting a large concentration of polymer-like CIAs (blue chains) surrounded by cations; such CIAs can displace $\mathrm{Cl}^{-}$ions within a Debye length, with normal $\mathrm{Cl}^{-}$concentration occurring elsewhere. An increase in cations and $\mathrm{Cl}^{-}$displacement in the immediate vicinity of membrane channels (but not away from them) could locally increase driving force for $\mathrm{Cl}^{-}$entry. The high charge density of CIAs, while providing a high average charge concentration value, corresponds to a relatively small fraction of the cytosol volume leaving large areas of unperturbed electroneutrality.

Figure 4. Possible physiological implications of electrodiffusion and electroosmosis in the synaptic cleft.

A: An AMPA receptor current generated shortly after release of glutamate (top, blue dots) gives rise to a strong flux of sodium ions (red dots) towards the cleft centre (red arrows), thus creating an electric field reaching $10^{3}-10^{4} \mathrm{~V} / \mathrm{m}$, thus accelerating glutamate escape (blue arrows). However, when receptor activation coincides with a postsynaptic action potential (middle panel), the flow of sodium ions (red dots) is directed away from the active AMPA receptor locations thus reversing the field. The latter temporarily retains glutamate in the cleft: in cerebellar synapses, this can boost activation of perisynaptic metabotropic 
receptors (middle panel) resulting in a potentiated NMDA receptor response (bottom) which in turn alters signal integration in the circuit ${ }^{48}$.

B: An AMPA receptor current generated shortly after release of glutamate (top) is carried by a strong flux of sodium ions towards the cleft centre (red arrows). The centripetal flow of sodium can exert both electrostatic and electroosmotic drag upon the charged sub-membrane ion layers including receptor proteins domains (top, green arrows). This could hypothetically prompt AMPA receptor accumulation towards the cleft centre, nearer to neurotransmitter release site (middle), thus leading to a potentiated synaptic current (bottom). 


\section{REFERENCES (key papers highlighted)}

1 Triller, A. \& Choquet, D. New concepts in synaptic biology derived from singlemolecule imaging. Neuron 59, 359-374 (2008).

2 Novak, P. et al. Nanoscale-targeted patch-clamp recordings of functional presynaptic ion channels. Neuron 79, 1067-1077 (2013).

3 Hochbaum, D. R. et al. All-optical electrophysiology in mammalian neurons using engineered microbial rhodopsins. Nat Methods 11, 825-833 (2014).

4 Tonnesen, J., Katona, G., Rozsa, B. \& Nagerl, U. V. Spine neck plasticity regulates compartmentalization of synapses. Nat Neurosci 17, 678-685 (2014).

5 Hodgkin, A. L. \& Huxley, A. F. A quantitative description of membrane current and its application to conduction and excitation in nerve. $J$ Physiol 117, 500-544 (1952).

6 Buzsaki, G., Anastassiou, C. A. \& Koch, C. The origin of extracellular fields and currents--EEG, ECoG, LFP and spikes. Nat Rev Neurosci 13, 407-420 (2012).

7 Pods, J., Schonke, J. \& Bastian, P. Electrodiffusion models of neurons and extracellular space using the Poisson-Nernst-Planck equations--numerical simulation of the intra- and extracellular potential for an axon model. Biophys $J$ 105, 242-254 (2013).

8 Mori, Y. \& Peskin, C. S. A Numerical Method for Cellular Electrophysiology Based on the Electrodiffusion Equations with Internal Boundary Conditions at Membranes. Communications in Applied Mathematics and Computational Science 4, 85-134 (2009).

9 Lopreore, C. L. et al. Computational modeling of three-dimensional electrodiffusion in biological systems: application to the node of Ranvier. Biophys J 95, 2624-2635 (2008).

10 Savtchenko, L. P., Korogod, S. M. \& Rusakov, D. A. Electrodiffusion of synaptic receptors: a mechanism to modify synaptic efficacy? Synapse 35, 26-38 (2000).

11 Zhang, L. I. \& Poo, M. M. Electrical activity and development of neural circuits. Nature Neuroscience 4, 1207-1214 (2001).

12 Eccles, J. C. \& Jaeger, J. C. The relationship between the mode of operation and the dimensions of the junctional regions at synapses and motor end-organs. Proc. R. Soc. Lond. B 148, 38-56 (1958). A pioneering theoretical study predicting a significant effect of electric fields on ion currents in small spaces such as the synaptic cleft.

13 Savtchenko, L. P., Antropov, S. N. \& Korogod, S. M. Effect of voltage drop within the synaptic cleft on the current and voltage generated at a single synapse. Biophys $J$ 78, 1119-1125 (2000).

14 Savtchenko, L. P. \& Rusakov, D. A. The optimal height of the synaptic cleft. Proc Natl Acad Sci U S A 104, 1823-1828 (2007).

15 Poo, M. M. Insitu Electrophoresis of Membrane-Components. Annu Rev Biophys Bio 10, 245-276 (1981).

16 Orida, N. \& Poo, M. M. Electrophoretic Movement and Localization of Acetylcholine Receptors in Embryonic Muscle-Cell Membrane. Nature 275, 31-35 (1978).

17 Eisenberg, B. Interacting ions in biophysics: real is not ideal. Biophys $J$ 104, 18491866 (2013).

18 Eisenberg, B. Ionic interactions are everywhere. Physiology (Bethesda) 28, 28-38 (2013). 
19 Holcman, D. \& Yuste, R. The new nanophysiology: regulation of ionic flow in neuronal subcompartments. Nat Rev Neurosci 16, 685-692 (2015).

20 Torriero, A. A. ed. Electrochemistry in Ionic Liquids: Volume 1: Fundamentals. . Vol. Volume 1: Fundamentals (2015).

21 Pods, J. A Comparison of Computational Models for the Extracellular Potential of Neurons. arXiv preprint arXiv:1509.01481 (2015).

22 Luo, Z. X., Xing, Y. Z., Ling, Y. C., Kleinhammes, A. \& Wu, Y. Electroneutrality breakdown and specific ion effects in nanoconfined aqueous electrolytes observed by NMR. Nat Commun 6, 6358 (2015).

23 DeFelice, L. J. Electrical properties of cells : patch clamp for biologists. (Plenum Press, 1997).

24 Steinberg, J. S. \& Mittal, S. Electrophysiology : the basics. Second edition. edn, (Wolters Kluwer Heath, 2017).

25 Macdonald, J. R. A New Model for the Debye Dispersion Equations. Phys Rev 91, 412-412 (1953).

26 Buyukdagli, S., Blossey, R. \& Ala-Nissila, T. Ionic current inversion in pressuredriven polymer translocation through nanopores. Phys Rev Lett 114, 088303 (2015).

27 Perram, J. W. \& Stiles, P. J. On the nature of liquid junction and membrane potentials. Phys Chem Chem Phys 8, 4200-4213 (2006).

28 Plonsey, R., Henriquez, C. \& Trayanova, N. Extracellular (volume conductor) effect on adjoining cardiac muscle electrophysiology. Med Biol Eng Comput 26, 126-129 (1988).

29 Clark, J. \& Plonsey, R. The extracellular potential field of the single active nerve fiber in a volume conductor. Biophys $J$ 8, 842-864 (1968). An important theoretical introduction to the use of mathematical formalism in calculating extracellular electric fields.

30 Thorne, R. G. \& Nicholson, C. In vivo diffusion analysis with quantum dots and dextrans predicts the width of brain extracellular space. Proc Natl Acad Sci U S A 103, 5567-5572 (2006).

31 Sykova, E. \& Nicholson, C. Diffusion in brain extracellular space. Physiol Rev 88, 1277-1340 (2008).

32 Hrabetova, S., Hrabe, J. \& Nicholson, C. Dead-space microdomains hinder extracellular diffusion in rat neocortex during ischemia. J. Neurosci. 23, 8351-8359 (2003).

33 Kinney, J. P. et al. Extracellular sheets and tunnels modulate glutamate diffusion in hippocampal neuropil. J Comp Neurol 521, 448-464 (2013).

34 Zheng, K. et al. Nanoscale diffusion in the synaptic cleft and beyond measured with time-resolved fluorescence anisotropy imaging. Sci Rep 7, 42022 (2017).

35 Rusakov, D. A. \& Kullmann, D. M. Geometric and viscous components of the tortuosity of the extracellular space in the brain. Proc Natl Acad Sci U S A 95, 89758980 (1998).

36 Hrabetova, S., Masri, D., Tao, L., Xiao, F. \& Nicholson, C. Calcium diffusion enhanced after cleavage of negatively charged components of brain extracellular matrix by chondroitinase ABC. J Physiol 587, 4029-4049 (2009).

37 Miranda, P. C., Hallett, M. \& Basser, P. J. The electric field induced in the brain by magnetic stimulation: A 3-D finite-element analysis of the effect of tissue heterogeneity and anisotropy. Ieee T Bio-Med Eng 50, 1074-1085 (2003). 
38 Bazhenov, M., Lonjers, P., Skorheim, S., Bedard, C. \& Dstexhe, A. Nonhomogeneous extracellular resistivity affects the current-source density profiles of up-down state oscillations. Philos Trans A Math Phys Eng Sci 369, 3802-3819 (2011).

39 Rusakov, D. A. Disentangling calcium-driven astrocyte physiology. Nature Reviews Neuroscience 16, 226-233 (2015).

40 Gleixner, R. \& Fromherz, P. The extracellular electrical resistivity in cell adhesion. Biophys J 90, 2600-2611 (2006).

41 Rudy, Y. \& Plonsey, R. Volume Conductor and Geometrical Effects on BodySurface and Epicardial Potentials .1. Theory. Physics in Medicine and Biology 25, 978-978 (1980).

42 Hallez, H. et al. Review on solving the forward problem in EEG source analysis. Journal of Neuroengineering and Rehabilitation 4 (2007).

43 Bagotsky, V. S. Derivation of the Main Equation of Gouy-Chapman Theory. (2006).

44 Mclaughlin, S. The Electrostatic Properties of Membranes. Annual Review of Biophysics and Biophysical Chemistry 18, 113-136 (1989).

45 Greathouse, J. A., Feller, S. E. \& Mcquarrie, D. A. The Modified Gouy-Chapman Theory - Comparisons between Electrical Double-Layer Models of Clay Swelling. Langmuir 10, 2125-2130 (1994).

46 Zheng, K., Scimemi, A. \& Rusakov, D. A. Receptor actions of synaptically released glutamate: the role of transporters on the scale from nanometers to microns. Biophys J 95, 4584-4596 (2008).

47 Nadler, B., Naeh, T. \& Schuss, Z. Connecting a discrete ionic simulation to a continuum. SIAM J. Appl. Math. 63, 850-873 (2003).

48 Sylantyev, S., Savtchenko, L. P., Ermolyuk, Y., Michaluk, P. \& Rusakov, D. A. Spike-driven glutamate electrodiffusion triggers synaptic potentiation via a Homerdependent mGluR-NMDAR link. Neuron 77, 528-541 (2013).

49 Guerrier, C. \& Holcman, D. Hybrid Markov-mass action law model for cell activation by rare binding events: Application to calcium induced vesicular release at neuronal synapses. Sci Rep 6, 35506 (2016).

50 Marhl, M., Brumen, M., Glaser, R. \& Heinrich, R. Diffusion layer caused by local ionic transmembrane fluxes. Pflugers Arch 431, R259-260 (1996).

51 McLaughlin, S. G., Szabo, G. \& Eisenman, G. Divalent ions and the surface potential of charged phospholipid membranes. J Gen Physiol 58, 667-687 (1971).

52 Ward, K. R., Dickinson, E. J. F. \& Compton, R. G. How Far Do Membrane Potentials Extend in Space Beyond the Membrane Itself? International Journal of Electrochemical Science 5, 1527-1534 (2010).

53 Stuart, G., Schiller, J. \& Sakmann, B. Action potential initiation and propagation in rat neocortical pyramidal neurons. Journal of Physiology-London 505, 617-632 (1997).

54 Bezanilla, F. The voltage sensor in voltage-dependent ion channels. Physiol Rev 80, 555-592 (2000).

55 Catterall, W. A. Ion channel voltage sensors: structure, function, and pathophysiology. Neuron 67, 915-928 (2010).

56 Neher, E. Correction for Liquid Junction Potentials in Patch Clamp Experiments. Method Enzymol 207, 123-131 (1992).

57 Lingane, J. J. Electroanalytical chemistry. (1958).

58 Dickinson, E. J., Freitag, L. \& Compton, R. G. Dynamic theory of liquid junction potentials. J Phys Chem B 114, 187-197 (2010). 
59 Barton, P. G. The influence of surface charge density of phosphatides on the binding of some cations. J Biol Chem 243, 3884-3890 (1968).

60 Gurtovenko, A. A. \& Vattulainen, I. Membrane potential and electrostatics of phospholipid bilayers with asymmetric transmembrane distribution of anionic lipids. J Phys Chem B 112, 4629-4634 (2008).

61 van Meer, G., Voelker, D. R. \& Feigenson, G. W. Membrane lipids: where they are and how they behave. Nature Reviews Molecular Cell Biology 9, 112-124 (2008).

62 Isaev, D. et al. Surface charge impact in low-magnesium model of seizure in rat hippocampus. J Neurophysiol 107, 417-423 (2012).

63 Rusakov, D. A. \& Fine, A. Extracellular $\mathrm{Ca}^{2+}$ depletion contributes to fast activitydependent modulation of synaptic transmission in the brain. Neuron 37, 287-297 (2003).

64 Annunziato, L., Pignataro, G. \& Di Renzo, G. F. Pharmacology of brain Na+/Ca2+ exchanger: from molecular biology to therapeutic perspectives. Pharmacol Rev 56, 633-654 (2004).

65 Marhl, M., Brumen, M., Glaser, R. \& Heinrich, R. Diffusion layer caused by local ionic transmembrane fluxes. Pflugers Arch 431, R259-260 (1996).

66 McLaughlin, S. G., Szabo, G. \& Eisenman, G. Divalent ions and the surface potential of charged phospholipid membranes. J Gen Physiol 58, 667-687 (1971).

67 Hahin, R. \& Campbell, D. T. Simple shifts in the voltage dependence of sodium channel gating caused by divalent cations. J Gen Physiol 82, 785-805 (1983).

68 McLaughlin, S. G., Szabo, G. \& Eisenman, G. Divalent ions and the surface potential of charged phospholipid membranes. J Gen Physiol 58, 667-687 (1971).

69 Hille, B., Woodhull, A. M. \& Shapiro, B. I. Negative surface charge near sodium channels of nerve: divalent ions, monovalent ions, and $\mathrm{pH}$. Philos Trans $R$ Soc Lond B Biol Sci 270, 301-318 (1975).

70 Isaev, D. et al. Role of extracellular sialic acid in regulation of neuronal and network excitability in the rat hippocampus. J Neurosci 27, 11587-11594 (2007).

71 Ednie, A. R. \& Bennett, E. S. Modulation of voltage-gated ion channels by sialylation. Compr Physiol 2, 1269-1301 (2012).

72 Michaluk, P. et al. Matrix metalloproteinase-9 controls NMDA receptor surface diffusion through integrin betal signaling. J Neurosci 29, 6007-6012 (2009).

73 Kochlamazashvili, G. et al. The extracellular matrix molecule hyaluronic acid regulates hippocampal synaptic plasticity by modulating postsynaptic L-type $\mathrm{Ca}^{2+}$ channels. Neuron 67, 116-128 (2010).

74 Dityatev, A., Schachner, M. \& Sonderegger, P. The dual role of the extracellular matrix in synaptic plasticity and homeostasis. Nat Rev Neurosci 11, 735-746 (2010).

75 Young, S. H. \& Poo, M. M. Topographical rearrangement of acetylcholine receptors alters channel kinetics. Nature 304, 161-163 (1983).

76 Qian, N. \& Sejnowski, T. J. An Electro-Diffusion Model For Computing MembranePotentials and Ionic Concentrations in Branching Dendrites, Spines and Axons. Biological Cybernetics 62, 1-15 (1989). A clear and detailed outline of the mathematical formalism of electrodiffusion pertinent to small spaces in the microenvironment of dendrites and synapses.

77 Langlands, T. A., Henry, B. I. \& Wearne, S. L. Fractional cable equation models for anomalous electrodiffusion in nerve cells: infinite domain solutions. J Math Biol 59, 761-808 (2009). 
78 Henry, B. I., Langlands, T. A. \& Wearne, S. L. Fractional cable models for spiny neuronal dendrites. Phys Rev Lett 100, 128103 (2008).

79 Halnes, G., Ostby, I., Pettersen, K. H., Omholt, S. W. \& Einevoll, G. T. Electrodiffusive Model for Astrocytic and Neuronal Ion Concentration Dynamics. Plos Computational Biology 9 (2013).

80 Halnes, G., Ostby, I., Pettersen, K. H., Omholt, S. W. \& Einevoll, G. T. An Electrodiffusive Formalism for Ion Concentration Dynamics in Excitable Cells and the Extracellular Space Surrounding Them. Advances in Cognitive Neurodynamics (Iv), 353-360 (2015).

81 Gianazza, E. \& Righetti, P. G. Size and Charge-Distribution of Macromolecules in Living Systems. Journal of Chromatography 193, 1-8 (1980).

82 Lodish, H. F. Molecular cell biology. 4th edn, (W.H. Freeman, 2000).

83 Glykys, J. et al. Local impermeant anions establish the neuronal chloride concentration. Science 343, 670-675 (2014).

84 Voipio, J. et al. Comment on "Local impermeant anions establish the neuronal chloride concentration". Science 345 (2014).

85 Kaila, K., Price, T. J., Payne, J. A., Puskarjov, M. \& Voipio, J. Cation-chloride cotransporters in neuronal development, plasticity and disease. Nature Rev. Neurosci. 15, 637-654 (2014).

86 Doyon, N., Vinay, L., Prescott, S. A. \& De Koninck, Y. Chloride Regulation: A Dynamic Equilibrium Crucial for Synaptic Inhibition. Neuron 89, 1157-1172 (2016).

87 Luby-Phelps, K. The physical chemistry of cytoplasm and its influence on cell function: an update. Mol Biol Cell 24, 2593-2596 (2013).

88 Luby-Phelps, K. Cytoarchitecture and physical properties of cytoplasm: volume, viscosity, diffusion, intracellular surface area. Int Rev Cytol 192, 189-221 (2000).

89 Leterrier, J. F. Water and the cytoskeleton. Cell Mol Biol (Noisy-le-grand) 47, 901923 (2001).

90 Fels, J., Orlov, S. N. \& Grygorczyk, R. The hydrogel nature of mammalian cytoplasm contributes to osmosensing and extracellular $\mathrm{pH}$ sensing. Biophys $J$ 96, 4276-4285 (2009).

91 Janmey, P. A., Slochower, D. R., Wang, Y. H., Wen, Q. \& Cebers, A. Polyelectrolyte properties of filamentous biopolymers and their consequences in biological fluids. Soft Matter 10, 1439-1449 (2014).

92 Verkman, A. S. Solute and macromolecule diffusion in cellular aqueous compartments. Trends Biochem Sci 27, 27-33 (2002).

93 Tuszynski, J. A., Portet, S., Dixon, J. M., Luxford, C. \& Cantiello, H. F. Ionic wave propagation along actin filaments. Biophys $J$ 86, 1890-1903 (2004).

94 Kekenes-Huskey, P. M., Scott, C. E. \& Atalay, S. Quantifying the Influence of the Crowded Cytoplasm on Small Molecule Diffusion. J Phys Chem B (2016).

95 Eccles, J. C. The physiology of synapses. (Springer-Verlag, 1964).

96 Poo, M. M. \& Young, S. H. Diffusional and Electrokinetic Redistribution at the Synapse - a Physicochemical Basis of Synaptic Competition. J Neurobiol 21, 157168 (1990).

97 Sylantyev, S. et al. Electric fields due to synaptic currents sharpen excitatory transmission. Science 319, 1845-1849 (2008). A first experimental demonstration of electrodiffusion phenomena affecting glutamatergic transmission in the synaptic cleft. 
98 Xie, Z. P. \& Poo, M. M. Initial Events in the Formation of Neuromuscular Synapse Rapid Induction of Acetylcholine-Release from Embryonic Neuron. P Natl Acad Sci USA 83, 7069-7073 (1986).

99 Groc, L. et al. Differential activity-dependent regulation of the lateral mobilities of AMPA and NMDA receptors. Nat Neurosci 7, 695-696 (2004).

100 Ashby, M. C., Maier, S. R., Nishimune, A. \& Henley, J. M. Lateral diffusion drives constitutive exchange of AMPA receptors at dendritic spines and is regulated by spine morphology. J Neurosci 26, 7046-7055 (2006).

101 Anantharam, V. et al. Combinatorial Rna Splicing Alters the Surface-Charge on the Nmda Receptor. Febs Letters 305, 27-30 (1992).

102 Choquet, D. \& Triller, A. The role of receptor diffusion in the organization of the postsynaptic membrane. Nat Rev Neurosci 4, 251-265 (2003).

103 Seeliger, C. \& Le Novere, N. Enabling surface dependent diffusion in spatial simulations using Smoldyn. BMC Res Notes 8, 752 (2015).

104 Constals, A. et al. Glutamate-induced AMPA receptor desensitization increases their mobility and modulates short-term plasticity through unbinding from Stargazin. Neuron 85, 787-803 (2015).

105 Czondor, K. et al. Unified quantitative model of AMPA receptor trafficking at synapses. Proc Natl Acad Sci U S A 109, 3522-3527 (2012).

106 Bliss, T. \& Lomo, T. Long-lasting potentiation of synaptic transmission in the dentate area of the anaesthetized rabbit following stimulation of the perforant path. $J$ Physiol 232, 331-356 (1973).

107 Ehlers, M. D., Heine, M., Groc, L., Lee, M. C. \& Choquet, D. Diffusional trapping of GluR1 AMPA receptors by input-specific synaptic activity. Neuron 54, 447-460 (2007).

108 Meier, J., Vannier, C., Serge, A., Triller, A. \& Choquet, D. Fast and reversible trapping of surface glycine receptors by gephyrin. Nat Neurosci 4, 253-260 (2001).

109 Cartailler, J., Schuss, Z. \& Holcman, D. Analysis of the Poisson-Nernst-Planck equation in a ball for modeling the Voltage-Current relation in neurobiological microdomains. Physica D-Nonlinear Phenomena 339, 39-48 (2017).

110 Cartailler, J., Schuss, Z. \& Holcman, D. Electrostatics of non-neutral biological microdomains. arXiv : 1612.07941 (2016). The most complete mathematical description to date of electrolyte electrodynamics in small cellular compartments.

111 Shilov, V., Barany, S., Grosse, C. \& Shramko, O. Field-induced disturbance of the double layer electro-neutrality and non-linear electrophoresis. Adv Colloid Interface Sci 104, 159-173 (2003).

112 Grienberger, C. \& Konnerth, A. Imaging Calcium in Neurons. Neuron 73, 862-885 (2012).

113 Rusakov, D. A. Disentangling calcium-driven astrocyte physiology. Nature Rev. Neurosci. 16, 226-233 (2015).

114 Bauer, M., Godec, A. \& Metzler, R. Diffusion of finite-size particles in twodimensional channels with random wall configurations. Phys Chem Chem Phys 16, 6118-6128 (2014).

115 Zitserman, V. Y., Berezhkovskii, A. M., Pustovoit, M. A. \& Bezrukov, S. M. Relaxation and fluctuations of the number of particles in a membrane channel at arbitrary particle-channel interaction. J Chem Phys 129, 095101 (2008). 
116 Mak, D. O. \& Webb, W. W. Conductivity noise in transmembrane ion channels due to ion concentration fluctuations via diffusion. Biophys J 72, 1153-1164 (1997).

117 Das, S. Electric-double-layer potential distribution in multiple-layer immiscible electrolytes: effect of finite ion sizes. Phys Rev E Stat Nonlin Soft Matter Phys 85, 012502 (2012). A theoretical investigation revealing how the size of ions can affects the electric field profile near charged cell membranes.

118 Bialek, W. \& Setayeshgar, S. Physical limits to biochemical signaling. Proc Natl Acad Sci U S A 102, 10040-10045 (2005).

119 Poo, M. \& Robinson, K. R. Electrophoresis of concanavalin A receptors along embryonic muscle cell membrane. Nature 265, 602-605 (1977).

120 Mclaughlin, S. \& Poo, M. M. The Role of Electroosmosis in the Electric-FieldInduced Movement of Charged Macromolecules on the Surfaces of Cells. Biophys $J$ 34, 85-93 (1981). A first study which shows experimentally and explains theoretically the effect of electro-osmosis on the lateral redistribution of membrane components.

121 Rusakov, D. A., Savtchenko, L. P., Zheng, K. \& Henley, J. M. Shaping the synaptic signal: molecular mobility inside and outside the cleft. Trends Neurosci 34, 359-369 (2011).

122 Linliu, S., Adey, W. R. \& Poo, M. M. Migration of Cell-Surface Concanavalin a Receptors in Pulsed Electric-Fields. Biophys J 45, 1211-1217 (1984).

123 Patel, N. B. \& Poo, M. M. Perturbation of the Direction of Neurite Growth by Pulsed and Focal Electric-Fields. J Neurosci 4, 2939-2947 (1984).

124 Henley, J. \& Poo, M. Guiding neuronal growth cones using Ca2+ signals. Trends Cell Biol 14, 320-330 (2004).

125 Dufreche, J. F., Jardat, M., Turq, P. \& Bagchi, B. Electrostatic relaxation and hydrodynamic interactions for self-diffusion of ions in electrolyte solutions. J Phys Chem B 112, 10264-10271 (2008).

126 Kobelev, V., Kolomeisky, A. B. \& Panagiotopoulos, A. Z. Thermodynamics of electrolytes on anisotropic lattices. Phys Rev E 68 (2003). 


\section{Acknowledgements}

This work was supported by the Wellcome Trust Principal Fellowship (101896), European Research Council Advanced Grant (323113-NETSIGNAL), Russian Science Foundation grant (15-14-30000, Fig. 1 data), FP7 ITN (606950 EXTRABRAIN).

\section{Competing interest statement}

The authors declare no competing interests.

\section{Still need:}

\section{TOC summary}

Neuroscience is going nanoscopic but can it still rely on classical electrophysiology? In this Review, the authors argue that accurate interpretation of physiological observations on the nanoscale has to account for electrodiffusion phenomena arising from local perturbations of ionic concentrations.

\section{Author biographies}

Leonid P. Savtchenko is a Senior Research Associate at the UCL Institute of Neurology, University College London, UK. Following his M.Sc. in theoretical physics, he obtained his $\mathrm{PhD}$ in theoretical neuroscience from Glushkov Institute of Cybernetics (Kiev, Ukraine), his D.Sc. in biophysics from Bogomoletz Institute of Physiology (Kiev), and received postdoctoral training at Dnepropetrovsk State University (Ukraine), CNRS (Marseilles) and International School for Advanced Studies (Trieste). His research focuses on various aspects of biophysical and computational neuroscience that can provide insights into the molecular, cellular, and network machinery underlying the empirically documented activity of central circuits.

Mu Ming Poo is the director, Institute of Neuroscience, Chinese Academy of Sciences in Shanghai, and an emeritus professor in UC at Berkeley. Having graduated in Physics at Tsinghua University, Taiwan, he obtained his $\mathrm{PhD}$ in biophysics from Johns Hopkins University. He was on the faculty of UC at Irvine, Yale, Columbia, and UC at San Diego, before joining UC at Berkeley, receiving multiple international scientific awards and prizes. Research in his laboratory focuses on cellular and molecular mechanisms underlying axon guidance, synapse formation and synaptic plasticity. Mu-Moing Poo's homepage http://www.ion.ac.cn/laboratories/int.asp?id=42.

Dmitri A. Rusakov is a professor and a Wellcome Trust principal fellow at the UCL Institute of Neurology, University College London, UK. Following his MSc in physics, he obtained his $\mathrm{PhD}$ in biophysics in Bogomoletz Institute of Physiology (Kiev, Ukraine) and received postdoctoral training at the Open University (Milton Keynes, UK) and at the National Institute for Medical Research, London. He has also received personal fellowship awards from the Medical Research Council and the Wellcome Trust. The laboratory he heads focuses on the principles of synaptic organisation and use-dependent plasticity, and also on 
the mechanisms of astrocyte-neuron communication. Dmitri A. Rusakov's homepage www.ucl.ac.uk/ion/synaptic-imaging. 


\section{Glossary terms}

Electrodiffusion is diffusion of charged particles in electric fields

Electrodynamic events: Time-dependent changes in electric fields or ion distributions

Averaging distance charasterises the scale over which a theoretical derivation or an estimate holds.

Debye length $L_{D}$ defines a scale over which the free charges, and therefore the electric field, are screened by an electrolytic solution.

Debye time is an average time required for an ion to move over Debye length.

Anisotropic media or fields display different properties in different directions, whereas the properties of isotropic media or fields do not depend on direction.

Second-rank tensor of conductivity is a $3 \times 3$ array (matrix) of values that characterise medium electrical conductivity in the $x, y$, and $z$ directions.

Dielectric media cannot conduct electric current.

Boltzmann distribution (sometimes called Gibbs distribution) is a probability distribution of the stochastically behaving particles being in a certain state.

Poisson-Boltzmann theory uses equations to describe the electrochemical potential of ions in the diffuse layer.

Continuum limit represents a theoretical approximation in which, at certain limiting scale, individual (discrete) system elements are considered as a continuous system's parameter or feature.

Van der Waals interactions are attractive or repulsive forces between molecules which are not related to (and is normally weaker than) covalent bonds or electrostatic forces. These may include dipole interaction, hydration, lipophilicity, etc.

Inner Hemholtz layer is formed in the sub-membrane space by cations that are attracted to the negatively charged cell membrane surface.

Gouy-Chapman theory of the electrical double layer provides classical formula to describe formation of diffuse charged layers occurring in the vicinity of a charged surface (membrane), due to free diffusion of small ions.

Monte Carlo models rely on computational algorithms that employ random number generation to mimic naturally occurring stochastic events, such as molecular Brownian motion.

Liquid junction potential (LJP) arises at a non-selective boundary between two electrolytes with different ion concentrations or mobility. 
Electrical double layer (EDL) is formed by free-diffusing electrolyte ions in the nanoscopic proximity of a charged surface, with the immediately adjacent layer of opposite-sign ions followed by a more diffuse layer of the same-sign ions.

$V^{*}$ potential represents the local electric field in the membrane proximity which drives voltage sensors of ion channels and other voltage-sensitive membrane proteins. Where exactly $V_{m}^{*}$ has to be measured is not clear and it may depend on the conformational features of individual proteins.

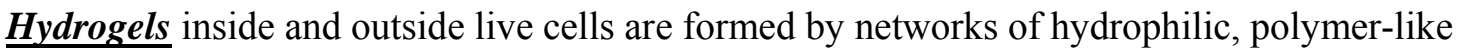
macromolecules often carrying surface change of a high density, with the flexible structure sensitive to the bulk $\mathrm{pH}$ and osmolarity.

Intracellular organelles are specialised subunits or multi-molecular complexes that are equipped with a specific function inside a cell.

Extracellular matrix (ECM) is a loose mesh, or possibly a hydrogel-like structure, comprising of fibrous proteins and polysaccharides that fill the interstitial space in the brain (and other tissues).

Sialylation is the biochemical reaction in which groups of sialic acid (a $N$ - or $O$-substituted derivative of neuraminic acid) are introduced into oligosaccharides and carbohydrates as the terminal monosaccharide. 
A

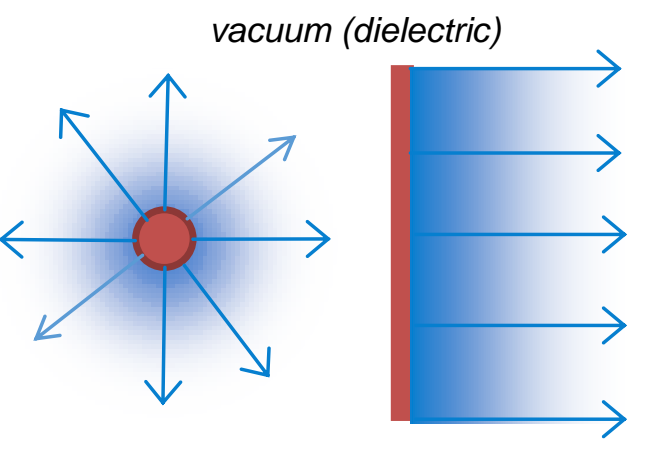

electrolyte

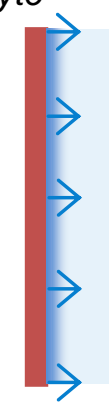

C

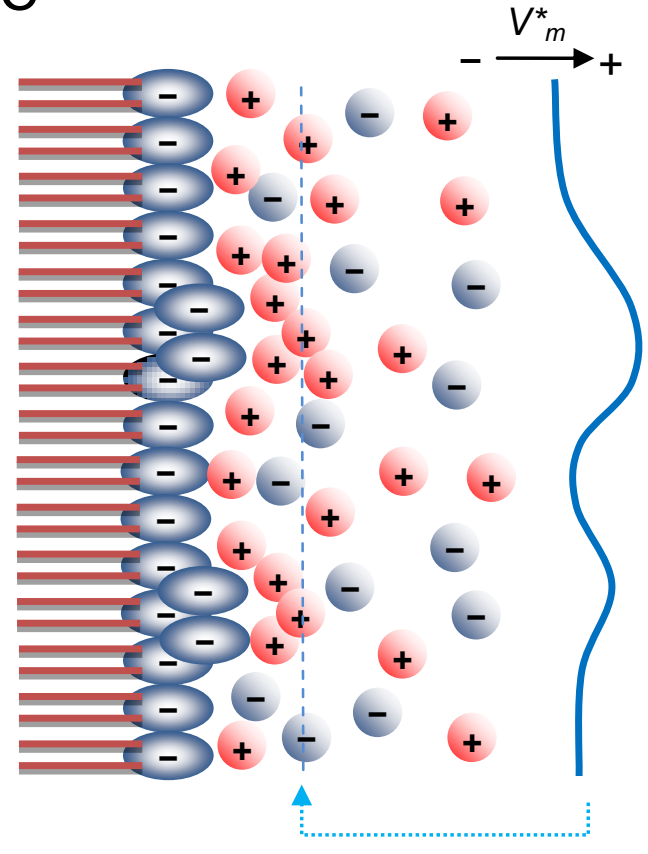

B

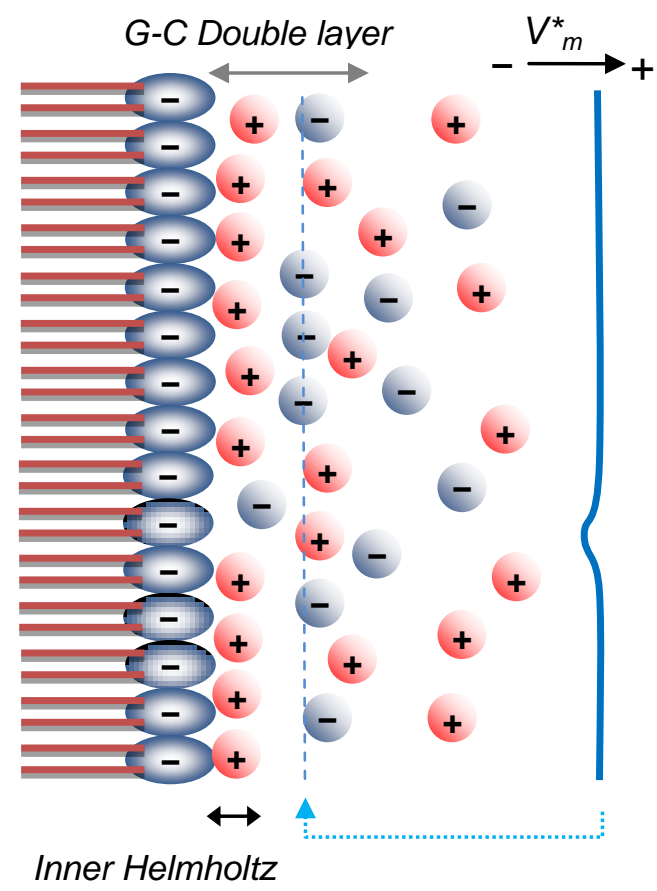

D

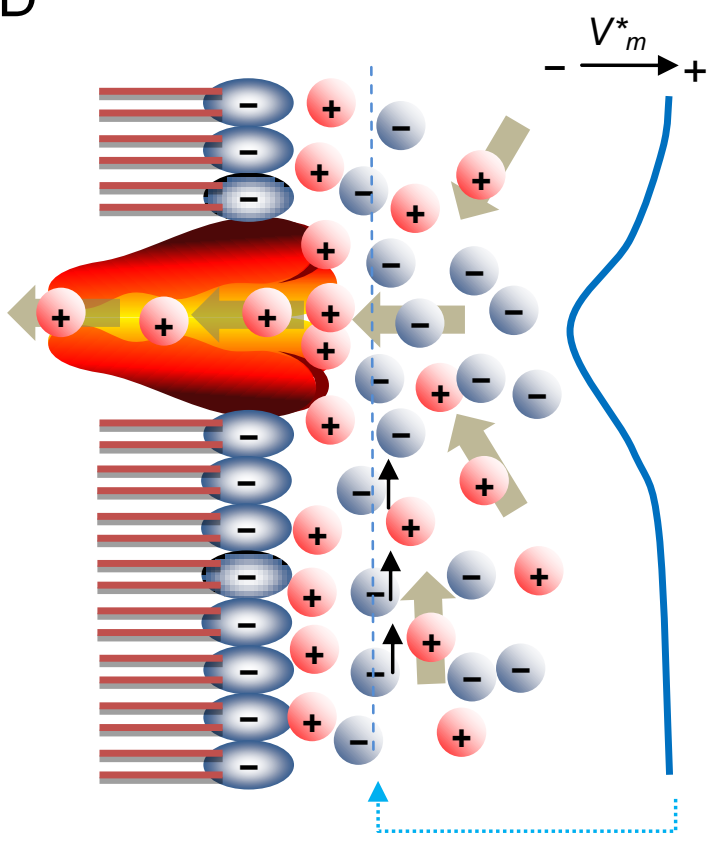

Figure 1 
A

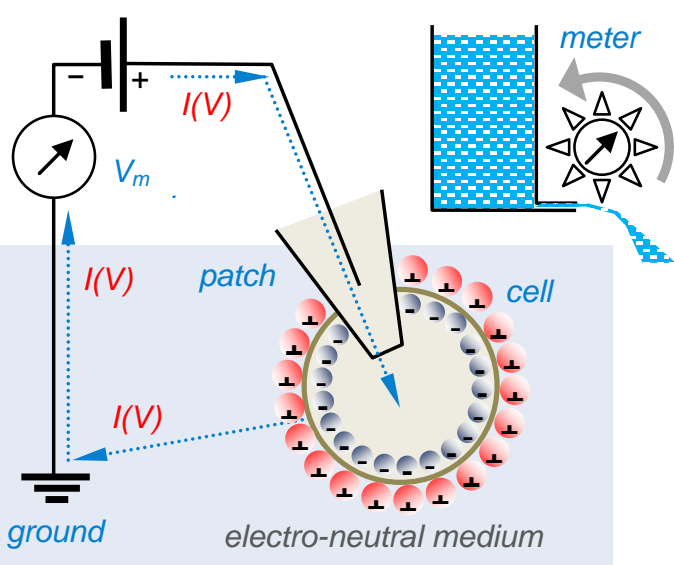

C Symmetric (canonical) case

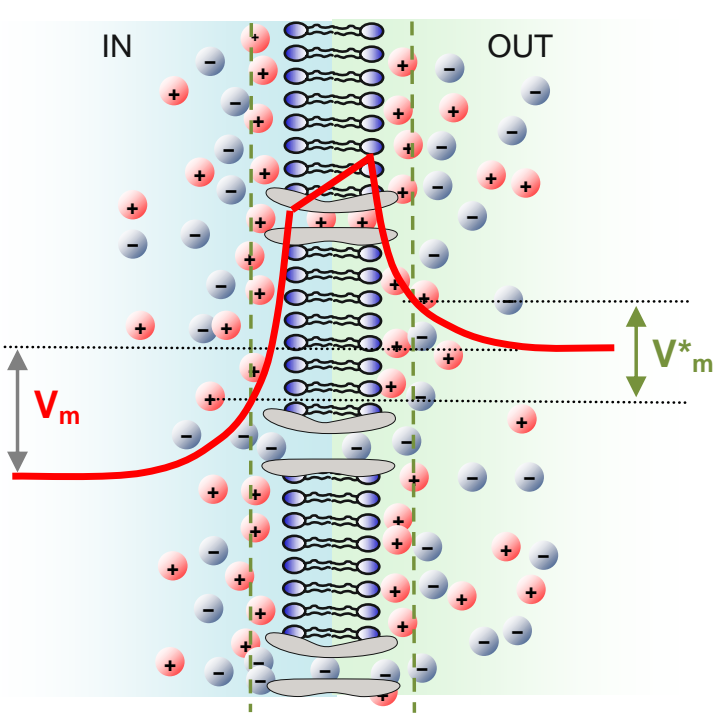

Divalent ion screening

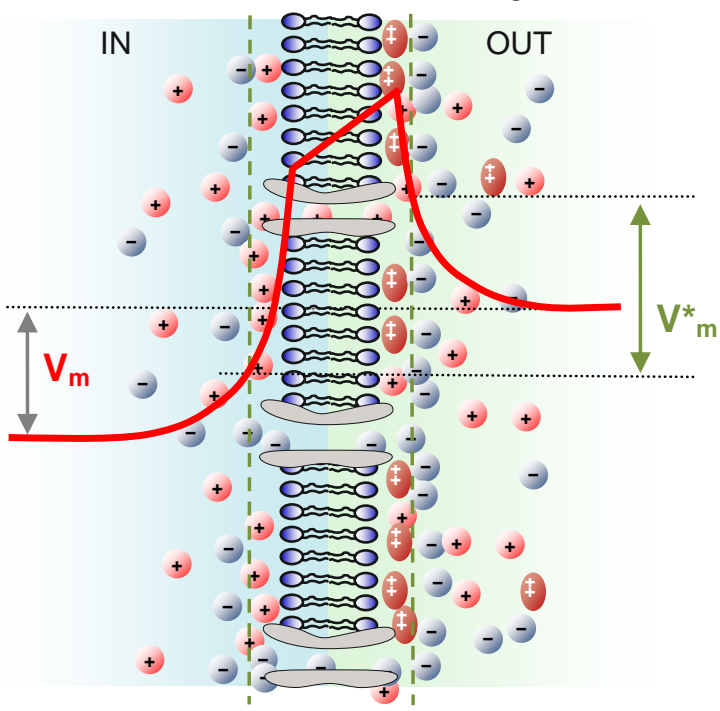

B

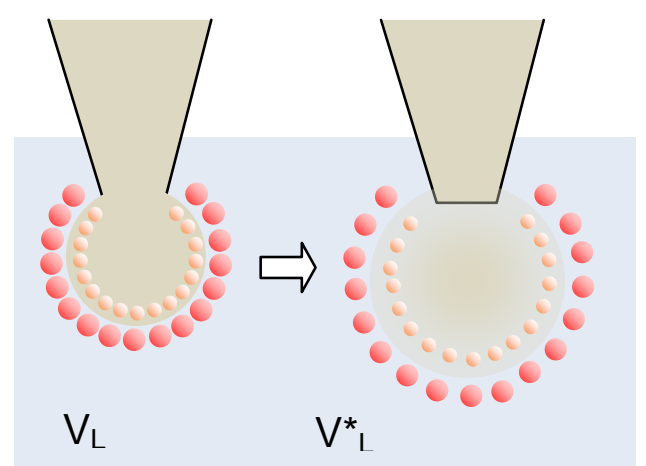

Asymmetric leaflets

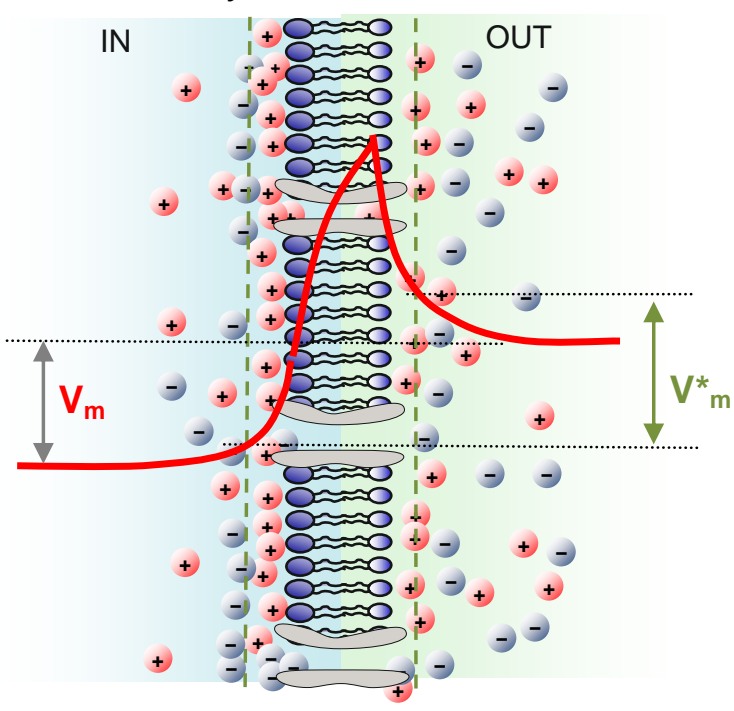

Neuraminidase charge cleavage

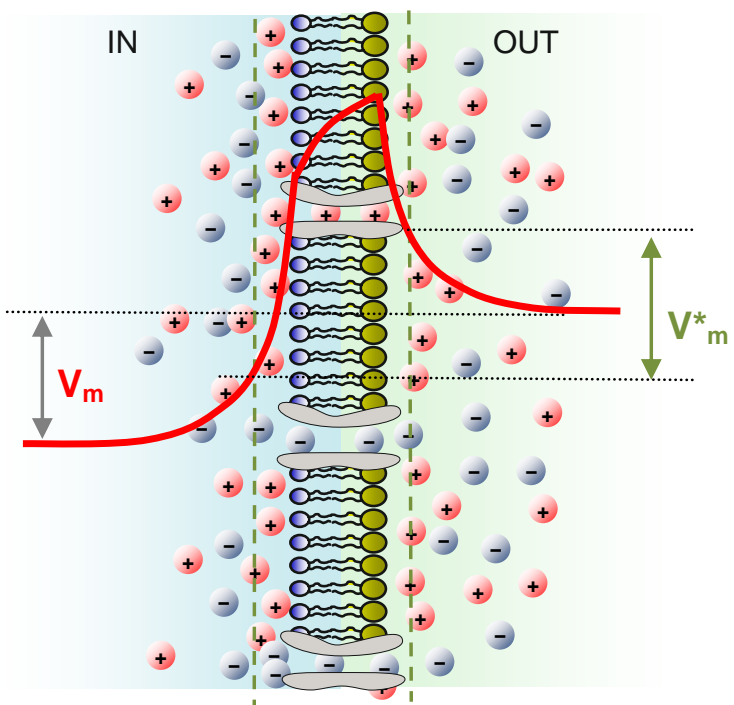

Figure 2 
A

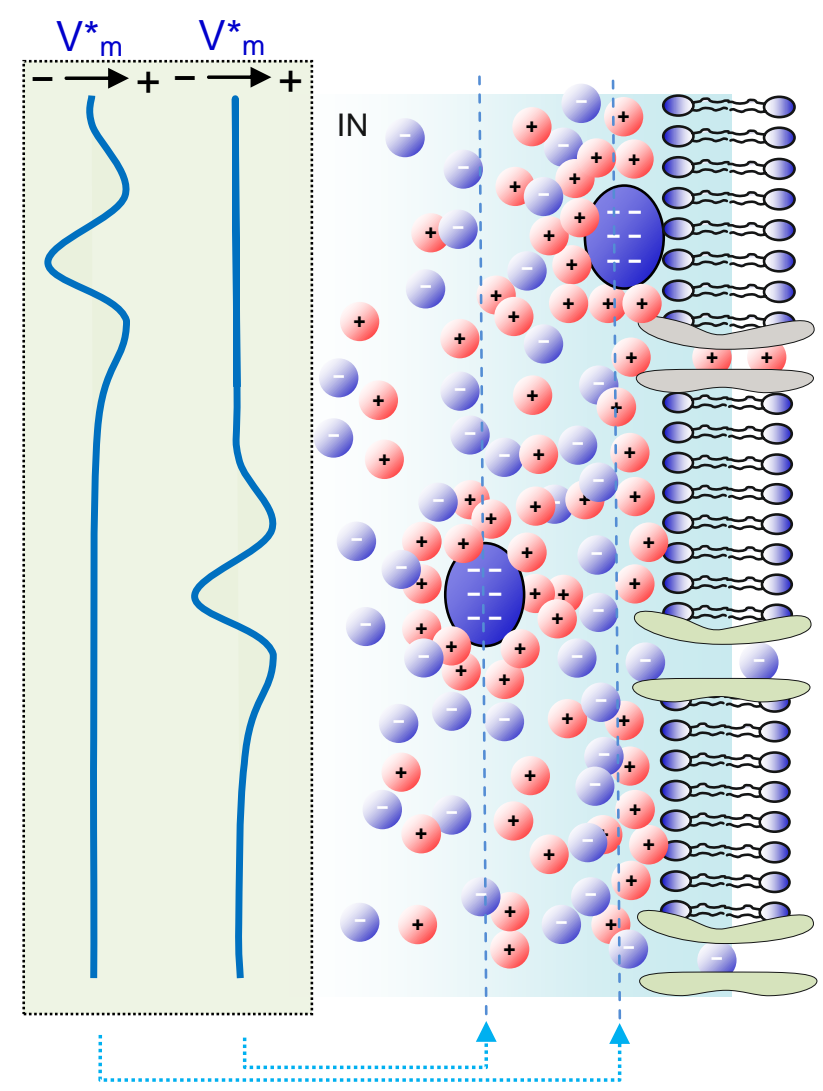

B

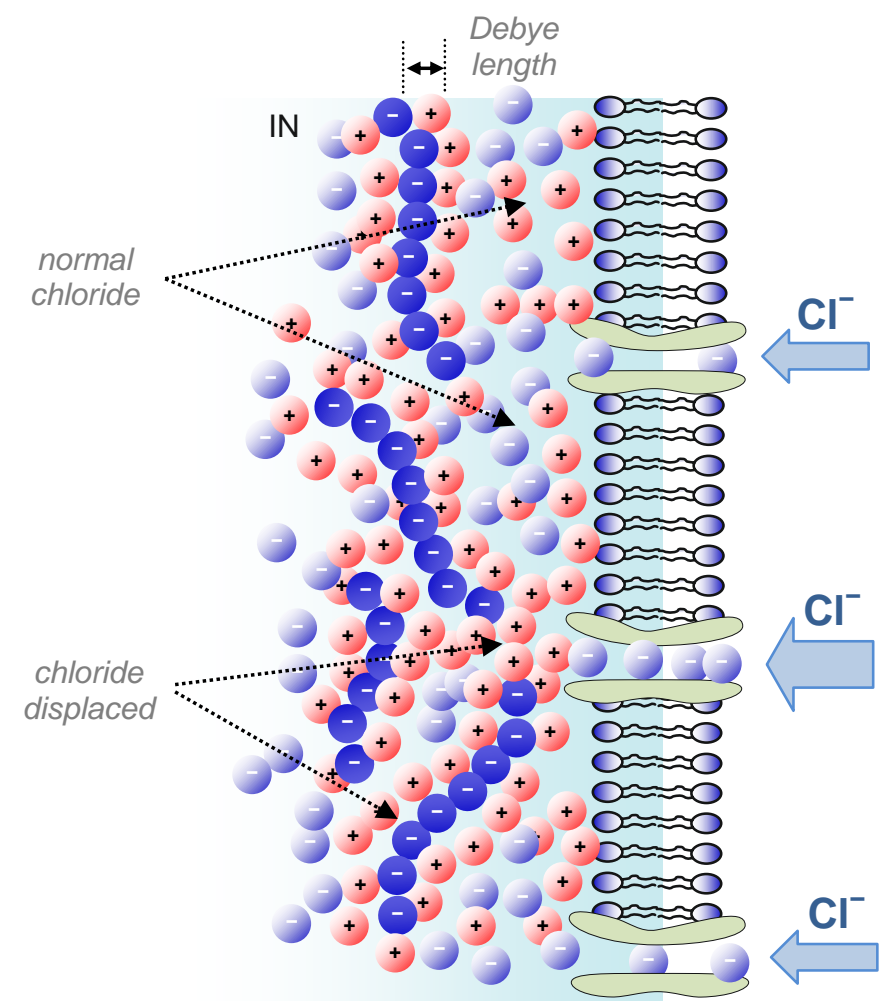

Figure 3 
A
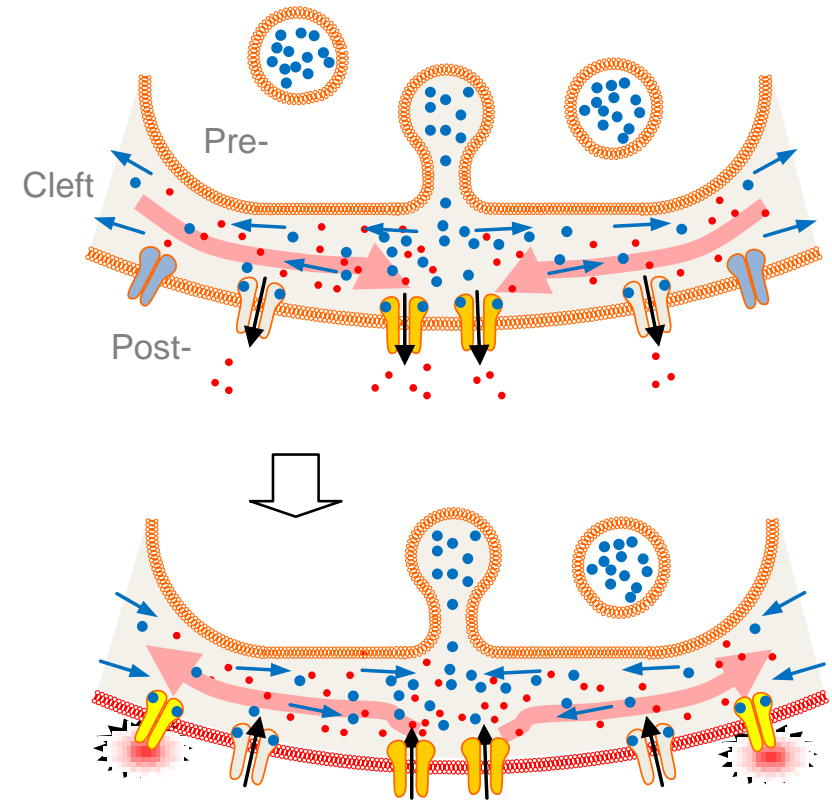

Postsynaptic spike

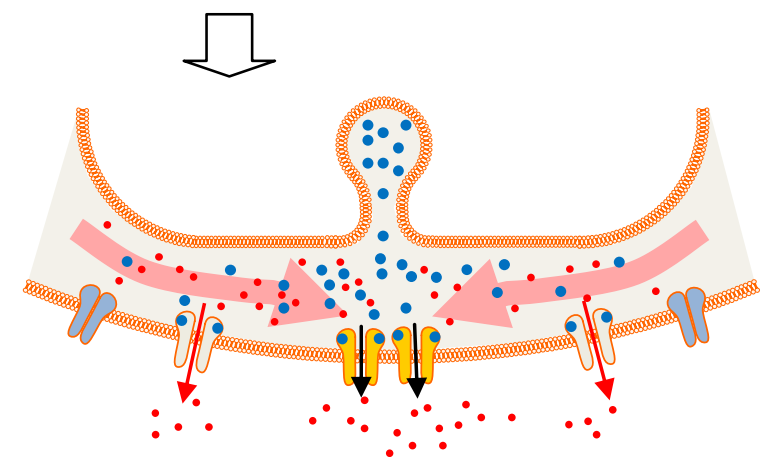

Potentiation
B
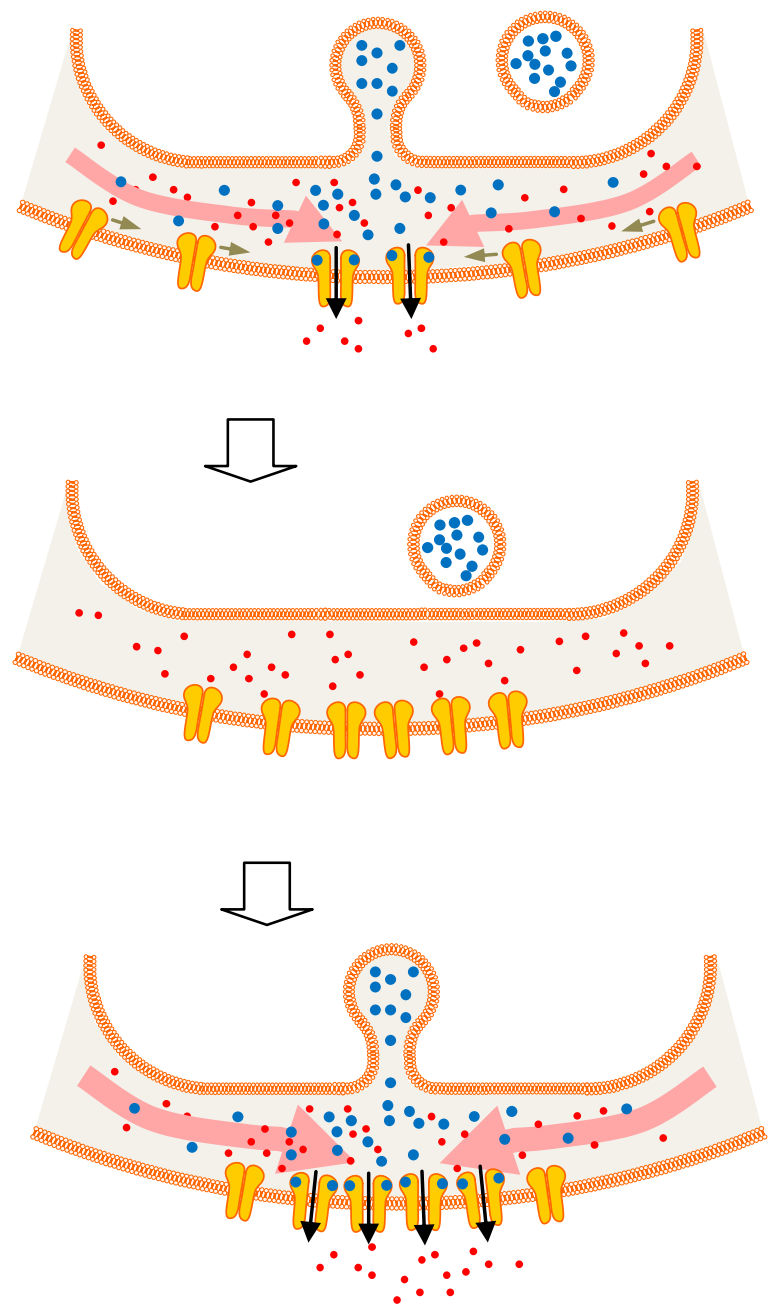

Potentiation

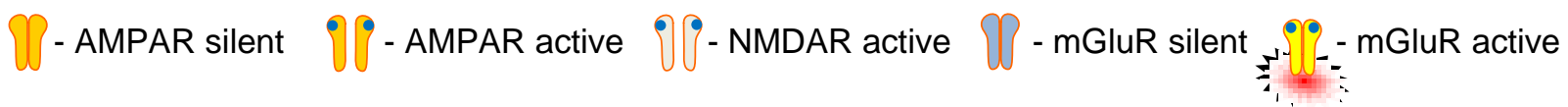

Figure 4 
A

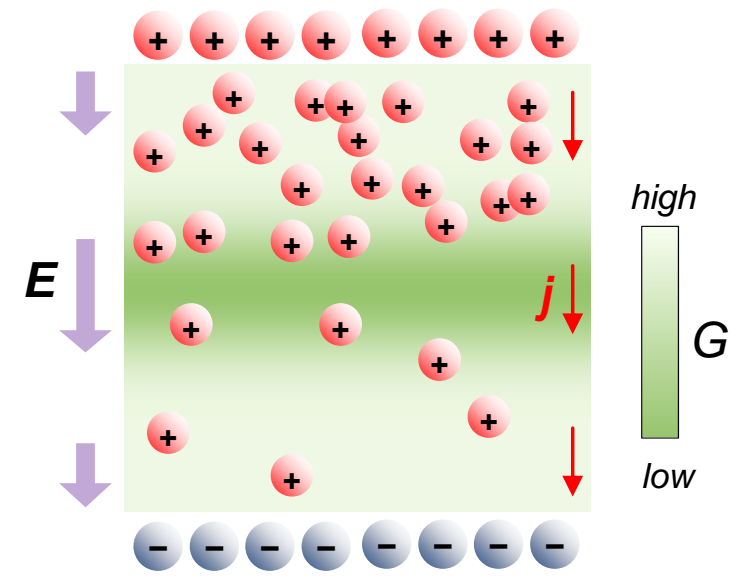

B

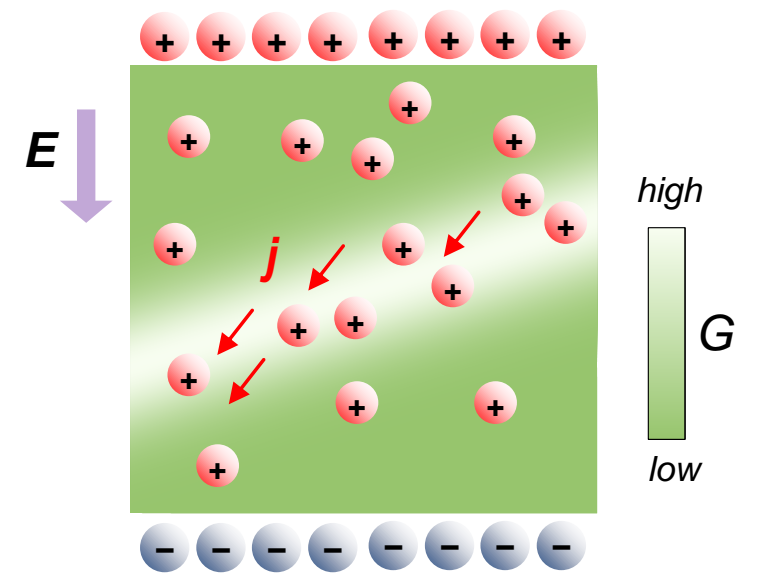

